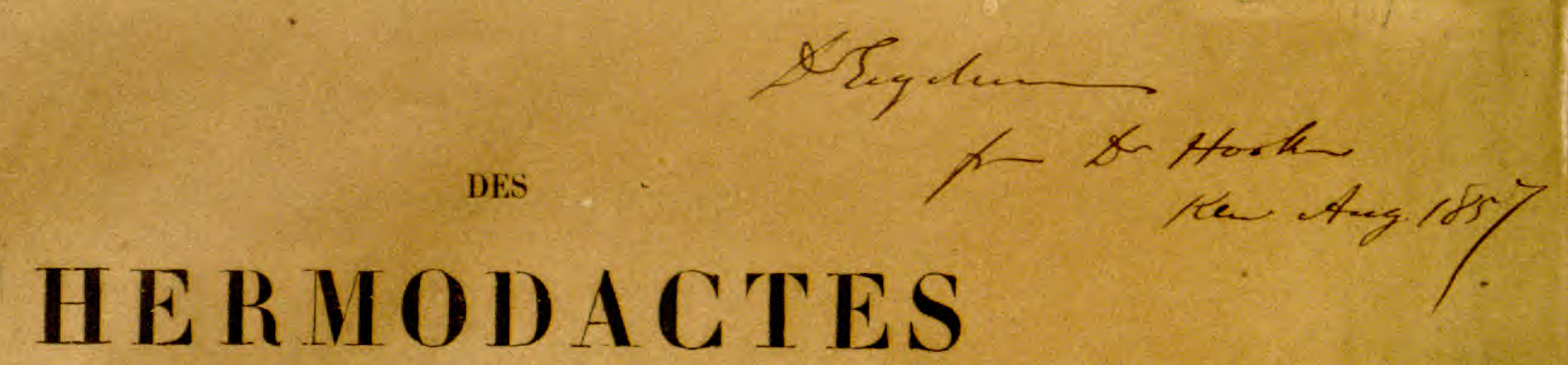

AU

POINT DE VUE BOTANIQUE ET PHARMACEUTIQUE.

\title{
THESE
}

PRÉSENTÉ ET SOUTENUE a L'ÉGOLE DE PHARMACIE dE PARIS,

Le 8 Janvier 1856 ,

PAn

\section{J.-E. Planchon,}

Docteur ès seiences et en médecine; Pharnacien; Professeur suppléant à la Faculté des sciences dẹ Monipellier.

" Medicus omnium stirpium, si fieri potest, peritiam habeat, consulo;

" sin minus, plurium saitem, quibus frequenter utimur. "

Galenvs, De Anlidot, Jib. 1.

\section{PARIS,}

IMPRIMERIE DE L. MARTINET,

2 , RUE MIGNON.

1856 


\section{DES}

\section{HER MODACTES}

AU

\section{POINT DE VUE BOTANIQUE ET PHARMACEUTIQUE.}

\section{THESE}

PRÉSENTÉe ET SOUTENUE a L'ÉGOle DE PHARMaGie de PARIS,

Le 8 Janvier 1856 ,

PAR

\section{J.-E. PLANGHON,}

Docteur ès sciences et en médecine; Pharmacien; Professeur suppléant à la Faculté des sciences de Montpellier.

"Medicus omnium stirpium, si fieri potest, peritiam habeat, consulo ; sin minus, plurium saltem, quibus frequenter utimur. „

Galenus, De Antidot, lib. I.

\section{PARIS,}

IMPRIMERIE DE L. MARTINET,

2, RUE MIGNON.

1856 


\title{
ÉCOLE SUPÉRIEURE DE PHARMAGIE.
}

\author{
ADMINISTRATEURS.
}

MM. Bussr, Directeur.

Guibourt, Secrétaire, Agent comptable.

Caventou, Professeur titulaire.

\section{PROFESSEURS.}

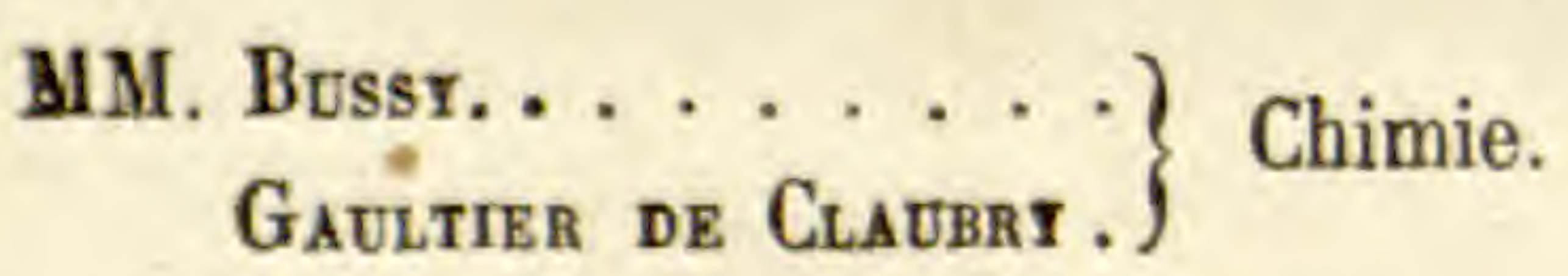

Lecand. . . . . . . Pharmacie.

Guibcut..... Histoire naturelle.

N. ..........

Chatin. . . . . . Botanique.

Caventort...... Toxicologie.

N......... Physique.
PROFESSEURS DÉLÉGUÉS

DE LA FACULTÉ DE MÉDECINE.

MM. WURTz.

Moquin-Taxdot.

\section{A GRÉGÉS.}

MM. Figuier, pour la chimie.

Robsquet, - la physique.

Reveir, - la toxicologie.

Luтz, - la pharmacie.

Souberrax, - l'histoire naturelle.

Nota. L'Ecole ne prend sous sa responsabilité aucune des opinions émises par les candidats. 


\section{J. DEC A IS N E,}

MEMBRE DE L'INSTITU T,

professeur de culture aU muséua d'histotre naturelle de paris, etc.

MoN CHER AMI,

Vous m'avez prêté pour cet opuscule vos conseils et votre érudition : c'est dire qu'il vous en revient la meilleure part. En le plaçant sous vos auspices, je double la valeur du reste. Si votre modestie souffre de cet hommage public, reportons-en l'honneur aux maìtres dont je suis heureux de recueillir auprès de vous les saines et solides traditions.

J.-E. PLANCHON. 


\section{DES \\ H E R M O D A G T E S}

$A U$

POINT DE VUE BOTANIQUE ET PHARMACEUTIQUE.

On désigne sous le nom d'Hermodactes, par contraction du mot grec Épuodóxтu入os, qui signifie doigt d'Hermès ou de Mercure, des tubercules ovoïdes-cordiformes, à peu près gros comme des châtaignes, un peu comprimés, convexes sur le dos, plus ou moins aplatis sur la face que parcourt un large sillon longitudinal peu marqué (fig. 1 et 2). Leur surface, d'une couleur ocreuse, est généralement lisse, rarement avec quelques traces de rides longitudinales : leur substance, homogène, compacte, légère, cassante et friable, se réduit aisément en une farine blanche, presque entièrement formée de fécule, et dont la saveur đouceâtre laisse à peine après elle une légère trace d'âcreté.

Longtemps célèbres dans la matière médicale comme un remède contre les affections articulaires, les Hermodactes sont tombés de nos jours en complète désuétude. On les conserve par habitude traditionnelle dans quelques vieux bocaux d'officine ou de droguier, où leur vétusté même, en les dépouillant de leurs propriétés actives, en fait d'ordinaire la pâture des insectes.

Une substance ainsi condamnée par la thérapeutique moderne pourra sembler peu digne d'une étude actuelle et rétrospective. Heureusement la seience a là-dessus des vies plus hautes que l'utilitarisme du jour; elle cherche la vérité pour elle-même, assurée d'ailleurs que l'utile est le fruit naturel du vrai : elle cultive l'érudition comme une justice due au passé et comme un excellent exercice pour l'esprit. Notre ambition sera satisfaite si nous ajoutons quelques arguments à l'appui de ces idées.

L'histoire des Hermodactes est encombrée de doutes et d'erreurs: doutes sur l'identité de cette substance chez les divers auteurs qui 
l'ont mentionnée : doutes sur la plante qui produit l'Hermodacte des officines. Il y a là toute une série de problèmes, dont l'étude sera l'objet de la première partie de ee mémoire; une seconde partie, plus spécialement botanique, sera consacrée à l'examen des tubercules du Colchicum variegatum, L., et de l'Hermodactylus tuberosus, Salisb. (Iris tuberosa, L.).

\section{PREMIĖRE PARTIE.}

HISTOIRE DES HERMODACTES.

\section{CHAPITRE I.}

HERMODAGTES VRAIS.

\section{$\S$ I. - Hermodactylos des Grees.}

L'Hermodactylos n'est mentionné ni chez Théophraste, ni chez Dioscoride, ni ehez Galien, ni chez aucun des premiers auteurs classiques de botanique médicale dont les ouvres sont parvenues jusqu'à nous. Alexandre de Tralles, célèbre médecin grec, qui florissait vers le milieu du $\mathrm{vi}^{e}$ siècle, signale le premier cette substance comme un remède précieux contre les maladies des articulations. Des passages que nous reproduisons en note (1) ressortent les faits suivants :

$1^{\circ}$ L'Hermodactylos agit comme purgatif.

2 . Il provoque des nausées et fatigue beaucoup les malades.

$3^{\circ}$ On l'administre à faible dose, associé tantôt à des purgatifs

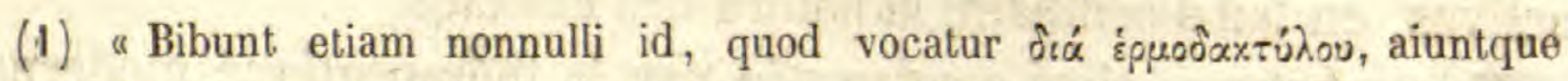
doloris levationem inde fieri, dum ex ventre quædam aquosa evacuat, ut etiam ægri statim inambulare velint. Atque hoc est verum, raroque id quod titulo promittit non evenit : sed habet etiam quod offendat, quoniam efficit, ut ii qui bibunt, crebrius fluxione irritentur. Hoc igitur ne fiat, nonnulli admiscuerunt cuminum, mastichen, zinziber : alii vero etiam euphorbium, arbitrati illud refrigeratorium quippiam et torporis inducendi efficax obtinere. Alii non adeo verum hoc esse dictitant : non enim ita purgare posset, si tantam refrigerandi facultatem haberet, ut torporem queat inducere. Sed falsum esse arbitror, omnes enim qui biberunt, eo die stomachum cibis, qui offerentur, non oblectari queruntur. Idonea igitur sunt quæ ex cumino, zinzibere et pipere participant, et malitiæ ipsius, quæ stomachum offendit, resistunt : sed nullum adeo ut aloe ipsi adjecta 
(Aloès, Scammonée, ete.), tantôt à des excitants aromatiques (Gingembre, Cumin, Anis, ete.).

\section{A part ces indications thérapeutiques et la signification du mot}

opitulari potest. Ego hujus compositionem et eorum, quæ veteres invenerunt, vobis exponam : ne ignoremus nos, neque ab aliis discere cogamur, sed is qui velit pro arbitrio uti, hic queat facile colligere.

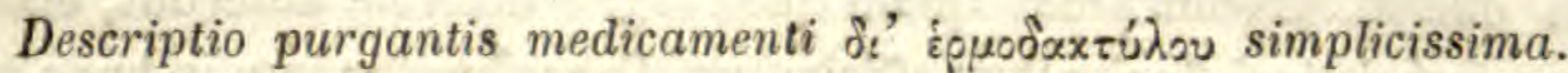

Hermodactyli drachma, gingiberis siliquæ novem, piperis siliquæ duæ, anisí totidem : hæc omnia una vice dantur. Sin autem ventrem copiosius subducere cogitas scammoniæ quatuor siliquas admisceto : et eitra molestiam purgat, ægrosque dolore levat.

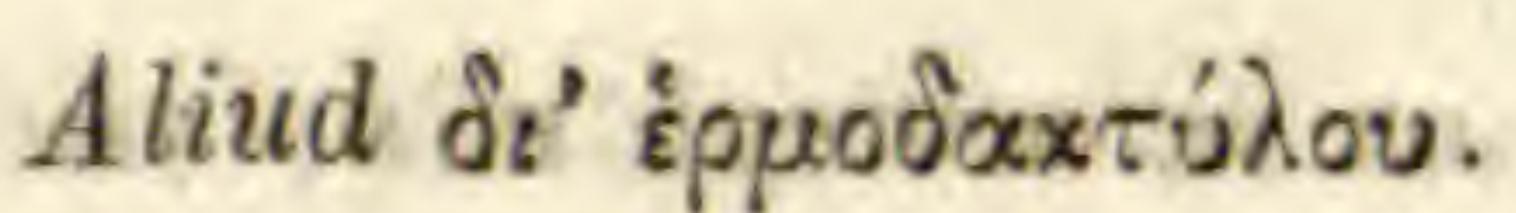

Hermodactyli unciæ tres, cumini scrupuli tredecim, gingiberis scrupuli dnodecim, piperis scrupuli decem : dantur scrupuli quatuor.

\section{Aliud ad idem.}

Hermodactyli scrupuli quatuor, gingiberis scrupuli sex, cumini siliquæ quatuor, piperis siliquæ quatuor, lacrymæ scammonii siliquæ octo. Dantur scrupuli quatuor ex mulso. Hoc medicamentum efficit, ut ægri statim incedant. Satius autem est ipsis dare morbo incipiente vel declinante.

Aliud valde bonum, experientia a nobis probatum.

Anisi, cumini, singulorum scrupuli 12, piperis albi et nigri, singulorum scrupuli 2, gingiberis scrupuli quatuor, euphorbii scrupuli quatuor, mastiches scrupuli sex, casiæ scrupulus, hermodactyli siliquæ quatuor. Hæc omnia una vice ex aqua temperata dantur.

\section{Aliud quod Jacobus Psxchrestus exhibebat.}

Hermodactyli scrupuli quatuor, scammonii scrupuli duo: dato potui ex aqua tepida, ubi ægrum victu prius commodo præparaveris.

\section{Egregium antidotum quod THEоротіus philosophus exhibebat.}

Hermodactyli siliquæ quatuor, cumini æthiopici scrupuli quatuor, anisi scrupuli tres, sii herbæ scrupuli sex, agarici scrupuli tres, musei siliqua, phu scrupuli tres, mastiches scrupuli duo, gingiberis scrupuli tres, spicæ nardi scrupuli duo, cnici scrupuli sex. Dantur scrupuli quatuor cum hydromelite. Præstantissimum est hoc medicamentum, ac plurimum a multis aliis medicis comprobatum. Oportet autem etiam ægrum victu prius præparare, atque ita duo vel tria ovorum lutea, panis exiguum et gallinæ pectus exhibere : deinde hora una interposita, aut multum duabus, ad balneum ducere : ac loto revertoque domum, ita antidotum ut prædictum est, debito pondere propinare. Hæc ratio victus in omni medicamento exhibendo utilis experientia apparuit. Neque enim hoc pacto medicamentum stomachum attingit, sed etiam ventrem longe facilius potest subducere; 
Hermodactylos, qui semble faire allusion à la forme d'une racine, aucun trait descriptif ne peut mettre sur la voie pour reconnaitre la nature et l'origine de la substance en question.

tantum in Hermodactylo nos experti sumus ac medicos nóstri temporis exhibere spectavimus : atque ego longo tempore his usus sum. Postea autem aloen ei admiscere utiliter, sum expertus, ac tentavi trochiscos potius fingere quam potionem offerre. Etenim præterquam quod ægris dolorem auferunt, etiam prohibent, ne crebris ipsi fluxionibus afficiantur. Describuntur ipsa in hunc modum.

Catapotia ex hermodactylo et aloe.

Aloes scrupuli duo, scammonii scrupuli quatuor, cucumeridis agrestis seminis semuncia, hermodactyli semuncia. Dantur scrupuli quinque aut sex pro viribus.

Aliud eadem efficiens, tum in articulariis, tum habitu pituitosioribus.

Aloes, colocynthidis, scammonii, singulorum semuncia : hermodactyli uncia, cum scrupuli aut sex : catapotia fingens, pro virium ratione dato. Quum autem emollientia alvum catapotia efficere voles, hoc pacto componito.

\section{Catapotia emollientia.}

Aloes siliquæ quinque, lacrymæ scammonii siliquæ septem, hermodactyli siliquæ tres, cucumeris agrestis seminis siliquæ tres. Omnia trita excipito rosato aut rhodomelo: fictaque catapotia dato, non modo podagricis, sed etiam aliis quorum alvum cogitas subducere. Omnia enim nihil adferent molestiæ, nihilque ingratum ac insuave quum devorantur, obtinent. »

Alexandi Tralliani opera lib. XI (de podagra), p. 309-310 du tom. I de la collection intitulée: Medicae artis principes post Hippocratem et Galenum, édit. Henri Estienne, ann. 1577.

Alexandre de Tralles naquit dans la ville de ce nom, en Lydie.

Haller pense qu'il dut visiter Rome et vivre peut-être en Egypte, et plus probablement encore en Phénicie.

Le chapitre spécial qu'Alexandre a consacré à la goutte est un des plus remarquables de son ouvrage. On voit qu'il traite cette maladie par les purgatifs. L'hermodactyle n'est jamais ordonné seul. 11 y adjoint des excitants aromatiques ou des purgatifs.

Outre les médicaments dans lesquels nous avons vu qu'il fait entrer l'hermodactyle, il emploie cette substance associée à la myrrhe, comme auxiliaire contre la goutte, suivant la formule suivante :

\section{Auxilium podagricum valde bonum.}

Myrrhæ siccæ sextula, hermodactyli sextulæ duæ : trita incretaque potui ex vino veteri in balneo exhibeto.

Ouvrage cité, édit. de Haller, Lausanne, 1772, in-8, vol. VII, p. 117. 
Paul d'Égine, plus connu sous le nom de Paullus Egineta, et qui vivait, probablement à Alexandrie, vers le milieu du vıe siècle, parle de l'Hermodactylos à peu près dans le même sens qu'Alexandre de Tralles (1). Il l'indique positivement comme une racine, et mentionne dans un autre chapitre de son ouvrage l'Ephemeron ou Colchicon de Discoride, espèce de Colchique, identique peut-être avec notre Colchicum autumnale (2), et depuis longtemps signalée comme une plante des plus vénéneuses.

D'après cette mention séparée faite par le même auteur du Colchicon et de l'Hermodactylos, Jean Ruellius, de Soissons (3), André Matthiole (4) et Adam Lonitzer (5), refusèrent d'admettre que l'Hermodactylos des Grees pût être le même que l'Hermodactyle des officines, confondu par les deux premiers avec le Colchique ordinaire ou Colchicum autumnale. Pour discuter en

(1) a Quidam vero in ipsis exacerbationibus (podagræ et arthritidis) in omnibus arthriticis purgatione ex hermodactylo utuntur : verum attendendum est, quia stomacho incommodus hermodactylus et anxios ac ciborum inappetentes facit. In solis igitur iis qui negotiis quæ differri non possunt, urgentur, ipso utendum est : compendio enim ef post duos ut plurimum dies sedat fluxionem, ut quis ad consuetas functiones obeundas transire possit. Novi autem quemdam qui non ipsam hermodactyli substantiam exhibet, neque aliquod medicamentum ex ipso compositum, sed herbam ipsam coquit, cum aliquo ex consuetis pharmacis, velut aniso, aut apio, et decoctum bibendum præbet, et mirum est quomodo per ventrem evacuati e fluxione liberantur. Oportet autem, propterea quod stomacho incommodus est (velut dixi) postea stomacho gratis et corroborantibus, cum moderata caliditate, osculum ventris corrigere. n

Paulli Egineta opera lib. VII, p. 495 A. C. dans la collection des Medica artis principes post Hippocratem et Galenum, édit. de Henri Estienne, 1567.

( Ermodactyli radix purgatoriam vim habet, tum ipsa tum decoctum ipsius. Privatim autem arthriticis in ipsis fluxionibus exhibetur: stomacho multum noxia est. 》

Paullus Egrneta, l. c. p. 620 H. 621 A.

(2) M. Fraas (Syn. Fl. class., p. 283) rapporte le Colchicon de Dioscoride au Colchicum variegatum; mais cette détermination spécifique ne repose sur aucune raison bien convaincante,

(3) J. Ruellius, De natura stirpium, lib. III, cap. cxv. Paris, 1536.

(4) A. Matthioli Comment. in Dioscor. (ed. Venet., 1565), p. 1109.

(5) A. Lonicerus, Botanicon (ed. Francof., 1565 ), p. 197. 
connaissance de cause la valeur de cette opinion, il faut exposer l'opinion contraire émise par les médeeins arabes.

\section{§ II. - Surugen ou Surengian des Arabes.}

Héritiers et souvent interprètes de la-science des Grecs, les Arabes nous ont laissé sur la matière médieale ancienne des renseignements utiles, bien qu'on ne puisse les accepter tous sans contrôle. Parmi les auteurs de cette nation, trois surtout méritent. d'être cités à l'occasion de l'Hermodacte.

Sérapion, dit le Jeune, que Sprengel suppose avoir vécu vers la fin du $\mathrm{x}^{\mathbf{e}}$ siècle de notre ère, regarde eomme identique l'Ephemeron ou Colchicon de Dioscoride, qu'il décrit, sans le nommer, d'après l'auteur grec, et l'Hermodactylos qu'il appelle en arabe Surugen (1).

(1) De Hermodactylis. CXCiIII. - Surugen id est hermodactylus, Dioscorides). Est herba quæ florescit in fine autumni et est ejus flos albus, similis in figura sua flori eroci, deinde emittit folia similia foliis bulbi, in quibus est humiditas adtrrens manui, et habet stipitem in longitudine unius palmi porrectum, cujus color est albus, declinans ad nigredinem et habet radicem cujus cortex est niger, ad rubedinem declinans. Sed quando aufertur cortex, apparet radix interius alba, mollis, dulcis saporis et plena humiditate. Est autem radix ipsa rotunda, similis cepe bulbi et habet radix ista in medio sui scissuram e qua egreditur stipes superquem est flos. Nascitur multum hæc herba in loce qui dicitur karin et in terris quæ dicuntur gnagna. Hæc herba si comedatur occidit strangulando, sicut occidunt fungi. Nos autem memoravimus esse illins in libro nostro ne errans aliquis comedat eum loco bulbi, nam ipsa est boni saporis, dulcis et delectabilis, quare fortassis comederet eam aliquis ignorans : conferunt autem illi, qui ipsum comederit, omnia quæ conferunt illi qui comedit fungos malos, est antem summum remedium ei lac bovinum, non egent namque ullo adjutorio qui eum sumpserunt. Et dixit iterum Dioscorides in capitulo de Achimeron et est lilium sylvestre, quod hermodactylus qui dicitur achimeron est interficiens et ita transtulit Albatarich in translatione sua, in medicinis simplicibus. Galenus). Habet virtutem laxativam et similiter aqua in qua bulierit et administratur in deloribus juncturarum. Paullus), Radix hermodactyli habet virtutem laxativam et similiter aqua in qua bulierit, datur autem proprie in doloribus juncturarum, in tempore fluxus humorum ad eas, verum ipse est malas stomacho. Bedigoras). Proprietas ejus est eonferre doloribus juncturarum. Damaschi). Confert dolori podagræe et est laudabilis finis operationis ejus, verum nimis usus e'us facit putrificari musculos. Confert 
Peu d'années après, le célèbre Abou-Ibn-Sina, nommé communément Avicenne, mentionne la même plante sous le nom de Surengian (1), mot qui se retrouve de nos jours, avec the légère

tamen juncturis multum. Et ideo exhibendus est maxime habentibus juncturas molles et nimis humidas, Ignotus). Surugen albus augmentum efficit in spermate. Mesarugiæ). Albus est bonus podagræ. Sed rubeus occidit et non est frigidusnimis, ita quod narcotizet quia si hoc esset non esset laxativus. Albugerig). Hermodactylus est calidus in primo gradu. Aben mesuai). Rubeus occidit et albus confert podagræet dessiccat hulcera antiqua, et abstergit ea, et est frigidus et siccus in secundo. Rasis). Si hermodactylus esset calidus, mordicaret ulcera. Alkalhemen). Hermodactylus est calidus, multæe caliditatis. Habix). Est calidus et siccus in principio secundi gradus, et habet proprietatem sedandi dolorem juncturarum et podagræe et conferre variolis et melior ex eo est ille qui est interius et exterius albus, et quando frangitur est confractio ejus facilis et non dura. Niger vero et rubeus sunt ambo interficientes. Erraverunt autem qui putaverunt quod ipse sit lagia agrestis, quæ defertur ab Aphrica, nam illa lagia impinguat mulieres, quando bibunt eam et humectat corpora sicca et augmentum efficit in carne et facit rubere faciem colore bono, quando datur in potu cum sanich, est calida, caliditate forti, et commoret sanguinem et acuit eum multum et fortassis indueet potanti se dolores ealidos, adustivos, sicut cholericos et sanguineos et infert nocumentum magnum, et ægritudines sicut erisipilam in capite et facie et in membris aliis corporis. $n$

SErapio, de temperamentis simplicium, p. 133, edit. curante Oth. Brunfelsio, in $-4^{\circ}$ sans date. (La dédicace porte la date de 4531 . Pas de nom d'imprimeur, ni de lieu dimpression.)

Tout ce passage de Sérapion est évidemment compilé des divers anteurs dont les noms y sont indiqués. Ce qu'il fait dire a Dioscoride, d'après la traduction d'Albatarich, relativement à l'Ephemeron (corrompu en Achimeron) est inexact, au moins par capport aux textes de l'auieur gree que nous connaissons, et dans lesquels il n'est pas question d'Hermodactylos. Ce mot n'est pas non plus daus les textes de Galien qui sont parvenus jusqu'à nous, et pourtant Serapion cite Galien avant Paul d'Egine, comme autorité pour les propriétés laxatives et antiarthritiques de l'Hermodactylos. D'ailleurs Sérapion brouille ensemble sous le nom d'Achimeron, I'Ephemeron vénéneux de Dioscoride, quiest un colchique, et l'Ephemeron non vénéneux du même auteur, plante encore indéterminée.

(1) De (Hermodactylo) Surengian.-Hermodactylus quid est? Est radix plantæ habentis rosas albas et citrinas. Et aperitur imprimis cum aperiuntur flores et oritur seu existit in inferiori parte montium et in eminentiis seu in collibus locorum planorum et folia ejus expansa sunt supra terram. Electio. Melior est albus exterius et interius durus ad frangendum. Et niger et rubeus sunt mali ambo. Natura. Calidus est et siccus, perveniens ad secundum. Et in ipso est 
altération (Sorinjan), appliqué par les Arabes aux Hermodactes qu'ils importent dans les bazars de l'Inde orientale (1). Le texte du passage d'Avicenne est probablement altéré, ou la traduction en est imparfaite, dans le passage où l'Hermodacte est indiqué comme produisant des Roses (fleurs) blanches et citrines. Aucun Colchique n'a des fleurs jaunes; or Avicenne entend parler d'un Colchique, aussi bien que Sérapion.

Un autre médecin arabe du $\mathrm{x}^{\bullet}$ siècle, Mésué (2), plus explicite au sujet de l'Hermodactyle, en distingue plusieurs sortes : d'abord une à racine longue comme le doigt; puis une à racine ronde,

humiditas superflua. Et existimaverunt quidam quod in albo est caliditas subtilis et in aliis virtus fortis : et si non non solveret. Et existimaverunt alii quod, si esset ealidus, tunc modicum ipsius mordicaret ulcera, sed in ipso non est mordicatio penitus. Et existimaverunt alii quod est calidus valde. Operationes et proprietates. Cum ipso est virtus solutiva, et si sit in ipso stypticitas. Vulnera et ulcera. Albus est bonus vulneribus antiquis. Instrumenta juncturarum. Confert podagræ et sedat dolorem statim, more emplastri superpositus. Et cum ex eo multototies sit emplastrum indurat apostema et in lapideam naturam convertit et est conferens theriaca omnibus doloribus juncturarum et proprie in oris fluxuum. Membra nutrimenti. Est malus stomacho debilitans eum : et rubeus et niger, retinent medicinas solutivas in stomacho et attrahunt nocumentum maximum. Membra expulsionis. In ipso est virtus solutiva et et efficit augmentum in coitu proprie eum zinzibere et mentastro et cymino. Venena. Rubeus et niger sunt venenum. Permutatio. Loco ejus ponitur in doloribus juncturarum pondus ejus de foliis alcannæ, et medietas ponderis ipsius de bdellio vario. » Avic. lib. II, cap. cccLr, pag. 247

Avicennz liber canonis, interprete Gerard. Carmonensi, édition annotée par André Alpagus et par Benoit Rinius, médecin de Venise.

Basileæ, 1558.

(1) Voyez Royle, Illustrat. of Bot., I, p. 387, et Pẹreira, Elem. of mat. med., ed. 3, vol. II, pars I, p. 1058.

(2) De hermodactylis. - Hermodactylus est radix herbæ cujusdam montanæ et ea vel digitum longa, vel rotunda : ac eadem præstantior, si multum est alba, et intus foris magna, mediocriter dura : altera scillæ aut raphano proxima, si crevit, si vere lecta est, et menses deinde sex sicca est. Rubra autem et nigra mala est, et quæ in loco pingui et humido provenit, quod inde humor ejus inflans et excrementosus ei largior insit, rara, levis, est imbecillior. Calidus est siccus ordinis secundi initio, cum humiditate tamen excrementosa, flatulenta, nauseabunda, quæ ventriculo nocet, etc., etc. (Suivent des détails sur les propriétés et 
laquelle comprend deux variétés, savoir: une blanche dont il recommande l'usage, et l'autre rouge et noire, qu'il déclare mauvaise. Nous essaierons bientôt d'établir la synonymie de ces prétendues racines.

\section{§ 3. - Hermodactes des officines.}

La forme et la structure de ces tubereules ne peuvent laisser douter un instant qu'ils ne proviennent d'une espèce deColchicum. Aussi rejetterons-nous au chapitre des faux Hermodactes, l'examen des plantes de divers genres auxquelles on les a souvent rapportés. La question du genre ainsi résolue, notre attention va se porter uniquement sur l'espèce de Colchique qui produit les vrais Hermodactes.

Nous avons vu Ruellius et Matthiole confondre avec les tubercules, vulgairement bulbes, du Colchicum autumnale, l'Hermodacte des apothicaires de leur temps, qui ne différait pas sans doute du nôtre. Cette erreur, partagée par Jérôme Tragus (1) et Leonhardt Fuchs (2), est implicitement réfutée par un passage de Valerius

l'administration du remède, détails pour lesquels liauteur compile Alexandre de Tralles et Paul d'Egine.)

Mesue opera (édit. de Venise, 1602 ), p. 57. Remèdes composés dans lesquels entre l'hermodactyle, mentionnés p. 165 (verso), du même ouvrage.

Le passage qu'on vient de lire est suivi d'un long commentaire de Johannes Costæus, d'où l'on peut extraire les observations suivantes :

L'hermodactyle d'A vicenne, de Sérapion et de Mésué, répond au colchique et à l'éphéméron de Dioscoride. Peut-être les Grecs donnaient-ils en commun à ces deux plantes le nom d'hermodactyle.

L'hermodactyle de Paul d'Egine n'est pas celui des Arabes, puisque cet auteur parle de l'éphéméron et de l'hermodactyle en deux chapitres différents. Mais, quelle est la plante du médecin grec? Question insoluble faute d'une description quelconque.

L'hermodactyle des officines n'est pas un médicament dangereux. A Venise e'est un remède de bonne femme. Perdrait-il par la dessiccation la plus grande partie de ses qualités nuisibles, et serait-ce pour cela que Mésué recommande celui qui est desséché depuis six mois ?

(1) H. Tragus, De stirp. comment. interprete D. Kibero, lib. II, cap. Lxrvı.

(2) L. Fuchsius, De hist. stirp. Lugduni, ann. 1551 , in-12, p. 351. 
Cordus (1), où l'un des caractères saillants de l'Hermodacte officinal, savoir sa couleur comparativement plus blanche que celle du tubercule de notre Colchique, est très nettement exprimé. L'absence à peu près totale de rides verticales chez l'Hermodacte, opposée à la présence de ces rugosités chez le Colchique ordinaire, établit entre les deux une distinetion non moins évidente. D'autres traits confirment cette diversité parfaitement mise en lumière dans les ouvrages classiques de Geoffroy, de Murray, de Pereira et de - M. Guibourt.

- Rembert Dodoens (2), en 1582, adopte les distinctions établies par Valerius Cordus entre l'Hermodacte blanc du commerce et le tubercule délétère du Colchique; il pense d'ailleurs que l'Hermodacte répond à l'Hermodactylos des médecins grees.

André Césalpin (3), en 1583, dit qu'on appelle vulgairement Hermodactylus un bulbe que sa deseription montre clairement être celui du Colchicum autumnale; mais il en distingue nettement l'Hermodacte médicinal ou du Levant (orientale), qu'il suppose être l'Hermodactyle à racine blanche et ronde de Mesué, tandis que l'Hermodactyle à racine ronde et noire, ou rougeâtre, du même auteur, serait notre Colchique ordinaire.

(1) De colchico albo (cujus radix etiam siccata, albicat tum foris, tum magis intus).

Colchicum album flore, foliis, semine el radice prædictis sirnile est, qualitate tantum ab illis differt. (Ces espèces précédentes, auxquelles l'auteur fait allosion sont : $1^{\circ}$ le Colchicum nigrum, cujus radice foris nigricat, et qui doit être notro C. autumnale; $2^{\circ}$ le Colchicum subrubens, quod a colore radicis ita cognominat, qui doit être une variétẻ ou espèce très voisine du précédent, avec lequel il croît mêlé); quanquam interdum flavescat, quum inveterascit, intus vero candidissima, tenera, in tundendo mitis, mollis et tractabilis existit: quæ tusa farinam frumentaceam adspectu refert. Saporem habet dulcem et valde suavem nulla amaritudine mixtum. Sponte in Germania nascentem nondum vidi, sed cultam tantum et e longinquis allatam locis.

VAberues Condes, Historiæ de Plantis, lib. II, cap. clmi, p. 463 (Ouvrage édité par les soins de Conrad Gesner, en 1564).

(2) R. Dodonæi Pemptades, lib. IV, cap. xxxm, La date que nous citons est celle que porte la préface de l'ouvrage, publié en 1616, après la mort de l'auteur.

(3) A. Cæesalpini de Plantis, lib. X, eap. xrx. 
Averti par ees observations, Jean Bauhin, dans son Historia plantarum, publiée en 1651 (1), rapportant avee détail les diverses opinions émises sur les Hermodactyles, appelle l'Hermodacte officinal Colchicum minus malignum sive Hermodactylus officinarum.

Vingt ans plus tard, en 1671, dans le Pinax theatri botanici de Gaspard Bauhin (2), ouvrage qui fut, jusqu'au temps de Linné, le code de la nomenclature botanique, l'Hermodacte figure avec sa synonymie sous le nom de Colchicum radice siccata alba.

Ces déterminations, on le voit, portent presque toutes sur le tubercule, tel que le présente le commerce, c'est-à-dire dépouillé de ses enveloppes, et séparé des organes qui pourraient servir à reconnaitre l'espèce qui le produit. Valerius Cordus, seul, semblerait avoir vu dans un jardin la plante vivante apportée de loin, et vaguement indiquée comme semblable au Colchique ordinaire par les feuilles, les fleurs, le fruit et la racine. Mais il est permis de douter que cet auteur ait eu sous les yeux la véritable plante à l'Hermodacte oriental, et qu'il ait décrit de ce dernier autre chose que le tubercule.

Par son talent de botaniste et son rôle dé voyageur en Orient, Tournefort était en mesure, mieux que personne, d'éclaircir cette question litigieuse. Malheureusement, ni ses ouvrages botaniques ni son herbier ne renferment aucun document à cet égard. Dans son Traité de matière médicale, publié après sa mort par Besnier, l'illustre auteur se borne à dire qu'il a souvent rencontré la plante à l'Hermodacte, dans l'Asie Mineure, avec les feuilles et les fruits du Colehique. Ce renseignement vague ne résout pas les doutes relatifs à l'espèce, mais il est important à recueillir comme preuve que l'Hermodacte existe dans l'Asie Mineure.

Dans tout ce qui précède, on ne trouve aucune détermination spécifique rigoureuse de la plante à l'Hermodacte, et l'on ne voit pas encore mentionné le Colchicum variegatum de Cornuti.

Un auteur anglais, Samuel Dale(3), est le premier, à notre con-

(1) Tom. II, p. 657-660:

(2) P. 67.

(3) S. Dałei Pharmacologia. Nous avons consulté l'édition in- $4^{\circ}$, publiée à Leyde, en 1739. 
naissance, qui nomme cette espèce comme étant peut-être la source de l'Hermodacte. Miller, dans son Gardener's Dictionnary (1), accueille avec réserve cette conjecture, et ne se prononce pas entre le Colchicum variegatum et son Colchicum tessulatum (Colch. Bivona, Guss.). M. Fée, en 1828 (2), adopte l'idée de Dale, qu'il attribue à contre-sens au vieux Matthiole. M. Geiger (3), en 1829, M. Kunze (4), en 1834, reprennent cette opinion d'une facon un peu dubitative. Ainsi fait Pereira (5), qui demande si l'Hermodacte ne proviendrait pas plutôt du Colchicum bulbocodioides, dont il sera question plus loin au chapitre des faux Hermodactes. Enfin M. Guibourt (6), en réfutant diverses erreurs de ses devanciers, et se prononçant nettement sur le genre de la plante à l'Hermodacte, hésite entre le Colchicum variegatum et le prétendu Colchicum illyricum d'Anguillara, plante imaginaire, que nous montrerons devoir disparaitre des catalogues de la science.

Ce rapide exposé de la question montre assez qu'elle est encore pendante, et que les livres ne suffisent pas à la résoudre. A défaut de l'observation directe de la plante dans le pays même où les tubercules se récoltent, nous avons cherché des renseignements dans les herbiers et les jardins.

Parmi les nombreux Colchiques que renferment les riches herbiers du Muséum de Paris, de M. Delessert, de M. J. Gay, de M. Cosson, aucun ne nous a semblé mieux répondre que le Colchicum variegatum aux Hermodactes du commerce. C'est la seule espèce du groupe des Colchiques, à fleurs automnales et précoces par rapport aux feuilles, dont les tubercules desséchés conservent une surface à peu près entièrement lisse, c'est-à-dire presque sans trace apparente de rides ou stries longitudinales. Ces stries sont, au contraire, manifestes chez le Colchicum autumnale, L., et les

(1) Nous consultons l'édition huitième, en deux volumes in-folio, de l'année 4768 .

(2) Hist. nat. pharm., I, p. 316-317.

(3) Handbuch der Pharmacie, p. 808.

(4) In Gobel's Waarenk., II, p. 270-271.

(5) Elements of mat. med., vol. II, part. 1, p. 1057-9, ëdit. 3, ann. 1850.

(6) Hist. des drogues, édit. 4, vol. II, p. 153-5. 
C.tessulatum, Mill. (C. Bivonce, Guss., C. latifolium, FI. Græe.), C. speciosum, Steven, C. Kotschyi, Boiss. (1), C. umbrosum, Stev., toutes espèces dont les tubercules peuvent se comparer à l'Hermodacte pour la grosseur. Nous laissons hors de comparaison la section des Colchiques dont les feuilles se montrent en même temps que les fleurs, par exemple le Colchicum bulbocodioides, parce que leurs tubercules dépassent rarement le volume d'une noisette, et n'atteignent jamais la grosseur moyenne des Hermodactes.

se Colchicum variegatum est parfaitement earactérisé dans le genre par ses feuilles ondulées, et ses fleurs roses rehaussées de panachures pourpres, dont la distribution en damier rappelle le

(1) Nous plaçons à côté de cette espèce une plante que M. Balansa vient de récolter dans le Taurus, et qu'il nous a bénévolement communiquée, sous le nom de $C$. candidum, Boiss. (Mais ce nom, indiqué sous toute réserve et de simple souvenir, ne se trouve pas dans les publications de M. Boissier que nous avons pu consulter.) La plante du Taurus est remarquable par l'extrême longueur du tube et par la forme très étroite des divisions de son périanthe; on peut la caractériser ainsi qu'il suit : $C$. Balanse, Planch.; autumnale, foliis hysteranthiis (ignotis), tubere ovato-cordato albo longitudinaliter striato, vaginis valde elongatis, floribus 6-8, perianthii tubo laciniis limbi circiter 5-plo longiore, laciniis lanceolato-linearibus acutiusculis 7-9-nerviis lilacinis vel albis, staminibus paulo infra faucem insertis majoribus perianthii limbi dimidium haud aquantibus, stylis stamina paulo superantibus apice leviter falcato acie interna longiuscule stigmaticis (vel si mavis stigmate lineari leviter recurvo).

Hab. Versant méridional du Taurus, au nord de Gulek-Boghas, en Cilicie; région montagneuse supérieure : Balansa, septembre 1855.

Cette espèee est très distincte d'une autre également récoltée par M. Balansa en Cilicie, à Gulek-Boghas, au nord de Tarsous, dans la région montagneuse inférieure. Cette seconde plante, plus voisine du Colchicum autumnale, présente des tubercules assez semblables aux précédents; mais ses gaînes florales et le tube de son périanthe sont beaucoup plus courts. Les divisions du limbe floral largement elliptiques et obtuses, à dix-neuf nervures au moins, n'ont pas tout à fait deux fois la longueur des étamines; les styles égalent presque la longueur de ce limbe, et les stigmates sont terminaux et punetiformes.

Les tubercules de ces deux espèces sont marqués de stries verticales, ce qui les distingue de ceux de ! Hermodacle; cependant il nous a semblé bon d'en parler, parce qu'ils pourraient bien se trouver mêlés çà et là à ceux de l'Hermodacte non strié. 
Fritillaria Meleagris (1). Les divisions acuminées de ces fleurs distinguent nettement l'espèce du Colchicum tessulatum, Mill., qui présente un coloris analogue, et sous lequel viennent se ranger comme simples variétés le Colchicum lusitanicum fritillaricum, et le Colchicum neapolitanum fritillaricum de Parkinson. Le même ancien auteur, dans son Paradisus, publié à Londres en 1629 , signala le premier le Colchicum variegatum sous le nom de Colchicum fritillaricum chiense. Cette belle espèce était, dès cette époque, ainsi que beaucoup d'autres du même genre, cultivée en Angleterre. Elle habite naturellement divers points de la Grèce insulaire et continentale, notamment les îles de Crète, de Chio, de Cos, ainsi que les environs de Smyrne, dans l'Asie Mineure. Nous n'en avons pas vu d'exemplaires provenant de la Syrie ou de l'Égypte.

Le tubercule de cette espèce n'offre, avons-nous dit, presque pas de traces de rides longitudinales : première et très importante coïncidence avec l'Hermodacte officinal. D'autres caractères complètent la ressemblance entre les deux tubercules vus dans le même état de dessiccation : forme, couleur de la surface et de la substance interne, forme des grains de fécule, saveur douceâtre avec un léger arrière-goût d’àcridité. Tout cela s'accorde assez pour faire eroire à l'identité d'origine des deux produits. Gardons-nous pourtant de présenter cette conclusion comme parfaitement décisive.

(4) Colchicum variegatur (Cornuti), L. - C. cormo lævi, foliis undulatis patentibus hysteranthiis, perianthii laciniis lanceolatis acuminatis acutis purpureo-tessellatis.

Colchicum variegatum (Cornuti). - L. Sp. pt. (édit. $3^{e}$ ), p. 484.- Lamk., Dict. II, p. 64. - Aiton, Hort. Kevo., édit. 2, vol. II, p. 330.-Redouté, Lil. tab. 238 (exclus. var. B?). - Bot. Magaz, t. 1028. - Rœem. et Schult., Syst., VII, p. 1507. - Trattin., Tabul. t. 240.

Colchicum fritillaricum chiense, the checkered Medowe Saffron of Chio or Scio, Parkins. Paradis., p. 456 et p. 155 f. 5. (ann. 4629).

Colchicum variegatum chiense, Gerarde, Herbal, p. 163, tig. 14 (ann. 1636). Colchicum variegatum, Cornuti, Canad, p. 136 et 137 : cum icone (ann. 1662). Colchicum chionense, floribus Fritillarice instar tessulatis, Moris., Hist., II, p. 234, sect. Iv, t. ui, f. 7 .

Colchicum fritillaricum chiense, Ray, Hist. $p l$.

Colchieum purpureum, magnis foliis viridibus, latioribus el erispis coum dictum, Tournef. Instit. 349 (fide floris unici in herb. Vaillant. ex hort. reg. paris.). 
Il faudrait, pour acquérir à cet égard une certifude complète, posséder des exemplaires d'Hermodacte officinal pourvus de leurs organes les plus caractéristiques (fleurs, fruits, feuilles), au lieu d'en être réduit à la simple étude du tubercule dénudé. Il faudrait aussi connaitre, d'une facon positive, les pays où l'on récolte ce produit, que les uns disent venir de l'Asie Mineure, les autres de Syrie ou d'Égypte, et toujours sans preuves que ces renseignements soient exacts.

Admettant done, mais ayec une certaine réserve, que l'Hermodacte dérive du Colchicum variegatum, nous allons tâcher d'établir la comparaison entre ce produit et l'Hermodactylos des Grees ou des Arabes.

§4. - Comparaison de l'Hermodacte des officines avec l'Hermodactylos des Grecs.

Au premier abord, rien ne parait plus difficile à concevoir que l'identité de ces deux substances médicinales. Comment retrouver, en effet, dans l'Hermodacte, la moindre analogie avec la forme d'un doigt, que le nom d'Hermodactylos semble supposer chez le tubercule ainsi désigné? Ceux-là même qui, se fiant à la tradition, reconnaissent dans l'Hermodacte actuel l'Hermodactylos des Grees, ceux-là, disons-nous, ont éludé plutôt qu'expliqué cette objection étymologique.

Plus favorisé par les eirconstances et par le recours à l'étude directe de la nature, nous allons faire de cetle objection une preuve, et de celte diffieulté la clef même de toute l'énigme. Constatons d'abord un fait important.

Le tubercule du Colchicum variegatum, probablement pareil en cela à d'autres espèces du genre, n'offre pas une forme constante. Tantòt it est ovoïde-cordiforme, au moins aussi large ou presque aussi large que haul, avec le processus inférieur sur lequel repose la pousse florifère beaucoup plus court que le corps même du tubercule. C'est ainsi que l'ont représenté Gerarde (Herbal, p. 163, fig. 14), Cornuti (Canad., p. 136), et plus tard Gawler dans le Botanical Magazine (tah. 1028). C'est ainsi que nous l'avons observé sur des exemplaires de l'herbier Delessert, et sur la plante 
vivante cultivée au jardin de l'École de médecine de Paris. Telle est aussi, sauf les nuances, la forme ordinaire des Hermodactes du commerce. D'autres fois, ce tubercule offre un corps principal ovoïde-oblong, avec un processus inférieur très allongé, qui présente l'apparence d'un ongle, ou, si l'on veut, de la phalange terminale d'un doigt. Cet état digitiforme du tubercule est celui que Parkinson a dû voir, lorsqu'il a décrit, dans son vieux et naïf langage, le Colchicum variegatum ou Colchicum fritillaricum chiense (1). On retrouve, bien longtemps après, la même forme figurée et décrite dans les Liliacées de Redouté ( tab. 238); enfin c'est elle que nous avons ici représentée (fig. 4) d'après un exemplaire vivant du Jardin des Plantes de Paris.

Si le mot hermodactylos, ou doigt de Hermès, semble peu convenir aux tubercules de Colchicum variegatum sous leur forme courte et ramassée, en revanche ce terme s'applique naturellement aux mêmes organes sous leur forme allongée et dactyloïde. Il y a dans ce rapprochement du mot et de la chose, à des siècles d'intervalle, un premier indice que vont confirmer des considérations d'une autre nature.

Les Hermodactes, tels qu'on les connait dans les droguiers et les officines, sont à peu près dépourvus de propriétés énergiques. C'est ce qu'ont prouvé les expériences de Gaspard Hoffmann et de

(1) Colchicum fritillaricum chiense. - The checkered Meadow Saffron of Chio or Scio.

. . The roote is like unto the others of this kinde but small and long and not so great : it flowered later for the most part than any of the other, even not until november, and is very hard to be preserved with us, in that for the most part the roote waxeth lesse and lesse every year, our cold country being so contrary unto his natural, that it will scarce shew his flower; yet when it flowreth any thing early, that it may have any comfort of a warme sunne, it is the glory of all these kindes.

Parkinson, Paradis., p. 156.

Dans les passages que nous avons soulignés, l'auteur constate que la racine (tubercule) est petite et longue, et que son volume diminue d'une année à l'autre, ce qu'il attribue à l'influence du climat trop froid de l'Angleterre. Nous présenterons plus loin, en parlant de la structure et du développement de ces tubercules, une autre conjecture sur la cause de ce fait. 
AU POINT DE VUE BotaniQue ET PHaRmaceutiQUe.

Van Swieten, citées par Murray (Apparat.medic., V, p. 219), et ce qu'explique l'analyse faite par L.-A. Lecanu, constatant dans cette substance l'absence de vératrine ou de tout autre alcaloïde. En supposant tout à fait exactes ces expériences thérapeutiques et chimiques, on aurait tort d'en inférer la nullité d'action des Hermodactes à l'état frais. Tout prouve, au contraire, que la dessiccation et surtout la vétusté altèrent la composition et débilitent l'action médicamenteuse des Colchiques, y compris le Colchicum autumnale. On a sur ce point le témoignage de Stœrck, qui, le premier, dans les temps modernes, établit sur des expériences directes l'emploi de ce dangereux remède. a In recenti radice, écrit le célèbre médecin de Vienne, sapor deprehenditur acerrimus, in annosa vero sapor fil farinaceus obtusus (1). "A l'égard du Colchicum variegatum, source probable de l'Hermodacte, le même fait peut aisément être constaté. Des tubercules de cette plante conservés dans l'herbier Delessert, sans avoir subi l'action du sublimé corrosif, nous ont offert une saveur douceâtre, en tout semblable à celle des Hermodactes; à l'état frais, au contraire, ces tubercules sont d'une odeur tout aussi vireuse, d'une saveur tout aussi âcre et amaricante que ceux du Colchicum autumnale. Leurs effets, selon toute apparence, ne seraient ni moins délétères à dose toxique, ni moins salutaires à dose médicamenteuse.

Ainsi s'expliquent, d'une part, le discrédit où sont tombés les Hermodactes vieillis des pharmacies, et, d'autre part, la confiance que ce remède a pu justement inspirer aux médecins grees qui l'avaient, en quelque sorte, sous la main, et pouvaient l'administrer à l'état frais. Nouvelle preuve que notre Hermodacte répond apparemment à l'Hermodactylos d'Alexandre de Tralles et de Paul d'Égine.

Rappelons, d'après le premier de ces auteurs, les propriétés de son Hermodactylos. C'est un remède purgatif ; ainsi du Colchique : on l'emploie contrể la goutte, et généralement les maladies articulaires; ainsi fait-on du Colchique. L'Hermodactylos fatigue et dégoûte les malades; le Colchique n'est pas moins redouté sous ce rapport. Bref, toutes ces coïncidences thérapeutiques semblent prouver au moins l'identité générique entre la plante à l'Hermo- 
dacte et l'Hermodactylos des Grees. Empruntons à la tradition un autre argument en faveur de cette idée.

\section{$\S 5$. - Comparaison de l'Hermodacte des officines avec le Surugen ou Hermodactylus des Arabes.}

En confondant sous un même ehef, et regardant à peu près comme équivalents l'Hermodactylos des Grees et l'Ephemeron de Dioscoride, Sérapion le jeune n'avait fait, sans doute, que recueillir dans l'école d'Alexandrie unc tradition véridique en somme, bien qu'un peu altérée dans les détails. Que Paul d'Égine ait consacré deux chapitres différents à l'Hermodactylos et au Colchicon, cela ne prouve rien contre l'identité générique des deux plantes. Il suffisait, pour expliquer cette mention séparée, que l'une eût été signalée comme remède, et l'autre comme un poison mortel. Les caractères botaniques ou de structure étaient aisément méconnus, lorsqu'ils auraient semblé contredire des caractères tirés des propriétés. Des analogies, vraies ou supposées sur ce dernier point, faisaient rapprocher sous le même titre les plantes les plus disparates, et, tout au contraire, la diversité présumée d'action médieamenteuse faisait assigner des noms différents à des plantes du même genre. Ainsi tombe l'objection soulevée par le vieux Matthiole contre l'identité générique de l'Hermodacte, de l'Hermodactylos , du Surugen, et de I'Ephemeron ou Colchicon.

Des trois sortes d'Hermodactyle ou de Surugen que signale brièvement Mésué, deux sont assez faciles à reconnaitre. La première, à racine longue, semble répondre à la forme dactyloïde du tubercule ci-dessus décrite chez le Colchicum variegatum, forme qui peut, du reste, se présenter chez d'autres espèces de Colchique. La seconde sorte, á racine ronde et blanche, est apparemment notre Hermodacte officinal, d'autant plus que Mésué parle spécialement de la racine desséchée depuis six mois, c’est-à-dire déjà singulièrement affaiblie dans son action. Avouons pourtant que la récolte de cette racine, indiquée pour le printemps (si crevit, si vere lecta est, etc.; voir ci-dessus, p. 12, note 2 , tout le passage), ne concorde pas avec la récolte probable des Hermodactes offieinaux; car ces derniers portent sur la face antérieure un sillon, qui 
ne se dessine que vers l'époque de la floraison, c'est-à-dire dans la dernière moitié de l'année. Or, au printemps, quand ce tubercule a poussé des feuilles, il doit être déjà bien flétri, et le nouveau tubercule ne doif pas avoir de sillon. Mais ces discordances n'empêchent pas de croire que le Surugen à racine blanche et ronde ne soit un Colchique, et peut-être le mêmeque le Sorinjan sheeran, ou Hermodacte doux des bazars de l'Inde orientale, que Pereira déclare identique avec I'Hermodacte des officines (1).

Il est difficile, pour ne pas dire impossible, de deviner à quelle espèce de Colchique se rapporte l'Hermodactyle à racine ronde, noire et rouge, de Mésué. Césalpin et Gaspard Bauhin ont cru y voir le tubercule de notre Colchique d'automne. Ceux-cirestent, en effet, souvent revêtus d'une pellicule fauve-rougeâtre (épiderme interne de la tunique foliacée qui les enveloppe), et pourraient passer pour rouges, tandis que la couleur d'un gris ou d'un rouge noirâtre qu'ils prennent souvent en se desséchant, expliquerait P'épithète de nigra. Mais tout cela n'est que simple conjecture,

(1) M. le professeur Boyle a rapporté des bazars du nord de I'Inde anglaise deux espèces de Sorinjan ou d'Hermodacte. Il suppose qu'elles sont importées de la mer Rouge par la voie de Surate ou de Bombay. La première espèce ést le Sorinjan sheeran, que nous venons de citer; la seconde, nommée Sorinjan tulkh, c'est-à-dire Hermodacte amer, est déerite comme il suit par Pereira, qui la rapporte avec doute à l'Hermodactylus rubeus et niger d'Avicenne et de Mésué.

« Les tubercules de cette variété se distinguent des précédents fc'est-à-dire de l'Hermodacte doux) par leur saveur amère, leur grosseur moindre, et par leur surface externe striée ou réticulée. Leur couleur est généralement plus foncée, noirâtre même chez quelques exemplaires. Un des tubercules est ovale-cordiforme, haut de $0^{\mathrm{m}}, 025$, large de $0^{\mathrm{m}}, 002$, sur une épaisseur d'environ $0^{\mathrm{m}}, 005$, canaliculé ou concave sur un côté, convexe sur l'autre, d'une teinte jaune brunâtre, demi-transparent et d'une texture très cornée. La coupe présente des lignes verticales qui semblent indiquer une texture lamellaire. Un second exemplaire est opaque, amylacé, réticulé à la surface externe, blanc à l'intérieur, moins aplati et d'une forme remarquable, le côté concave du tubercule se prolongeant de près de $0^{\mathrm{m}}, 045 \mathrm{au}$-dessous du point d'attache des anciennes fibres radicales. D'autres tubereules sont de la grosseur et de la forme de pepins d'orange, mais aplatis ou creusés sur un côté; quelques-uns sont vermoulus. Un autre offre extérieurement une couleur brun-noirâtre. "(Traduit de Pereira, Elements of mat. med., éd. 3, vol. II, pars 1, p. 1058.) 
et la vérité sur ce point n'aurait d'ailleurs que peu d'importance.

§ 6. - Conclusions des précédents articles.

Un fait capital ressort des considérations qui précèdent : c'est l'identité probable de l'Hermodactylos des médecins grees du $\mathrm{vi}^{e}$ et $\mathrm{vII}^{\mathrm{e}}$ siècle avec notre Hermodacte, et conséquemment avec une espèce de Colchique, soit le $C$. variegatum, soit une autre espèce orientale.

C'est pour avoir contesté ce fait, et récusé sans raison le témoignage des Arabes sur cette question de matière médicale, que la thérapeutique a laissé dormir des siècles entiers la découverte des propriétés des Colchiques dans le cas de maladies articulaires. L'étude des plantes n'est donc pas aussi stérile pour la médecine que l'on affecte de le croire : l'érudition elle-mème n'est pas toujours un luxe inutile, une prétentieuse superfluité; enfin on peut, sans paraitre trop sévère, rappeler à la jeunesse studieuse le respect que la science moderne doit aux maitres des anciens temps.

\section{CHAPITRE II.}

FAUX HERMODACTES.

Les produits les plus divers ont usurpé le nom d'Hermodacte, et, sur cette litigieuse question, on est surpris de voir d'étranges erreurs sanetionnées par l'autorité des noms les plus respectables. Indiquons rapidement ces faux Hermodactes, en commencant par ceux qui se rapprochent le plus du vrai.

\section{§1. - Colchicum autumnale.}

Parmi les botanistes de la Renaissance, Ruellius, Matthiole, Tragus et Fuchs ont pris à tort l'Hermodacte officinal pour le tubercule du Colchique d'automne. II est probable que beaucoup d'apothicaires de l'Europe oceidentale faisaient la même confusion, au grand détriment des malades. Plus récemment, des médecins distingués sont tombés dans cette erreur, mais en usant du Colchique avec toute la prudence requise. De ce nombre sont 
M. Want (1), qui, dès 1815, indiqua le premier l'efficacitè du Colchique dans le traitement des maladies rhumatismales (2), et sir William Halford, président du Collége des médecins de Londres (3) : ce dernier avait pourtant pris la précaution de faire venir des Hermodactes du marché de Constantinople, et les avait soumis à ses collègues, qui ne surent les distinguer de ceux du Colchique ordinaire, Il nous semble inutile de revenir sur cette distinction évidente, déjà bien établie plus haut sur les données de Valerius Cordus, de Césalpin, de Gaspard Bauhin, de Geoffroy, et de la plupart des auteurs de matière médicale.

\section{§ 2. - Hermodactes d'Égypte.}

Colchicum bolbocodioides, M. Biebst. Fl. taur.-caucas.

Colchicum agyptiacum, Boissier, Diagnos. n V, p. 66 (ann. 1844) (4).

(1) J. Want, in Med. and Physic. journal, vol. XXXII, d'après Pereira.

(2) J. Want, Ibid., vol. XXXIII et XXXIV, d'après J. Kuhn, Dissert. sur les propriétés méd. des Colchicacées, Strasbourg, 1827, p. 22.

(3) W. Halford, Lond. med. Gazette, vol. VIII, p. 318.

(4) Colchicum egmptiacum Boiss. - Espèce fondée sur la plante récoltée en Égypte, dans le désert voisin d'Alexandrie, par Aucher-Éloy ( $n^{\circ} 2155$ de sa collection). Nous en avons sous les yeux les exemplaires authentiques, ainsi que d'autres parfaitement semblables, recueillis aux mêmes lieux par M. Kralik (Herb. du Muséum). Les divisions florales ne sont pas nues à la base, mais bien munies d'une double crête assez longue et très étroite. Ces divisions' sont comparativement beaucoup plus étroites que dans le Colchicum bulbocodioides; mais ce caractère ne parait pas assez important pour distinguer ces plantes comme espèces.

Chez d'autres exemplaires incomplets, récoltés sur les bords de l'Euphrate par le colonel Chesney, les divisions florales sont plus étroites encore, au point de pouvoir être dites linéaires. On n'y voit pas clairement des crêtes internes: mais il est plus que douteux qu'on puisse séparer la plante du $C$. bulbocodioides.

D'après ce qui précède, on voit que la présence de crêtes sur les divisions du périanthe, n'a pas assez de constance pour servirà caractériser un sous-genre dans le type Colchicum. Mais on pourrait très bien admettre cette section nommée Hermodactylus par Rob: Brown, en y comprenant non plus seulement, comme a fait Kunth, le Colchicum Ritchii, mais toutes les espèces dont les feuilles naissent en même temps que les fleurs. Une objection se présente cependant, à l'adoption du mot Hermodactylus pour cette section des Colchiques à feuilles synanthes, c'est que le même terme est adopté génériquement pour I'Iris tuberosa. 
Colchicum Ritchii, Rob. Br. in Oudn. Denh. and Clappert. p. 36 (ann. 1826); fide cl. Cosson.

Colchicum hololophum, Coss, et Dur, exsiccat., monente cl. Cosson (1). Colchicum montanum, Forsk. Fl. aggypt.-arab., p. 77.

Prosper Alpin, dans son ouvrage sur la médecine des Égyptiens (De medic. Egypt., lib. III, p. 234) (2), dit que les femmes de ce

(1) Le Colchicum hololophum, Coss, et Dur., que M. Cosson a rapporté luimême comme synonyme au Colchicum bulbocodioides, M. Biebst. (voir Cosson et Kralik, Catal. des pl. de Syr. et de Pal. récoltées par MM. de Saulcy et Michon, p. 19), paraît être, au premier abord, une espèce très distincte. Il est remarquable surtout par la largeur des divisions ae son périanthe, dont les extérieures sont elliptiques, presque obovales et obtuses au sommet. Toutes ces divisions portent à la face interne, vers leur base, deux crêtes saillantes, étroites à bord généralement cuuni d'une ou deux dents. Ses feuilles, plus courtes que les fleurs, sont coriaces, un peu arquées, pliées en gouttière, rudes sur les bords, à cause de petites aspérités clair-semées.

M. Cosson a comparé ses exemplaires avec un échantillon authentique du Colchicum hulbocodioides étiqueté par M. Steven, et conservé dans l'herbier Webb. Il a reconnu l'identité des deux plantes l'une avec l'autre, ainsi qu'avec le Colchicum Ritchii de M. Rob. Brown, dont il a vu un exemplaire provenant de Tripoli.

Les échantillons $n^{o s} 5263,5369$ et 2158 bis, récoltés dans l'Asie Mineure par le voyageur Aucher-Éloy, s'accordent avec ceux du C. hololophum d'Algérie, sauf que leurs divisions florales sont plus étroites et plus aiguës.

(2) a Sed ex omnibus pro secreto habent singulo die, dum eunt dormitum, ad decem vulgares bulbos, pro Hermodactylis a nostris pharmacopæis receptos; quos aliqui potius Colchicum esse autumant, contostos mandere, eosque pluribus diebus, quindecim scilicet et viginti ad usque, frequentant. Ex quorum usu, quod nostris mirum videbitur, nihil vel per alvum vel vomitum evacuantes, minusque aliqua molestia mulieres vexantur. „

\section{Remarque du commentateur Guilandinus.}

Hinc nostros falsis uti Hermodactylis, cognoscitur, dubiumque etiam fit, eos esse Dioscoridis Colchicum, cum per os sumpti nullam noxam inferant, minimeque strangulent, Nisi loci diversitate illi collecti aliam a nostris facultatem obtineant, minimeque venenosam.

Second passage du meme ouvrage, lib. IV, p. 253

" Mulieres pauperculæ sumptum pro aliis ferre nequeuntes, vulgares Hermodactylos, quibus communiter nostri pharmacopolæ utuntur, modice contostos æque 
pays mangent souvent, avant d'aller se coucher, jusqu'à dix bulbes d'Hermodacte, qu'elles font rôtir à la façon des Châtaignes. Ainsi préparés, ces bulbes n'agissent nullement comme un purgatif, et contribuent, avec les bains et d'autres conditions de régime, à procurerà ces femmes l'embonpoint qu' elles recherchent comme un signe de beauté. L'auteur ne distingue pas ces bulbes des Hermodactes que l'on vendait de son temps dans les officines d'Italie. Mais s'il est vrai que les tubercules dont les Égyptiennes faisaient usage, fussent indigènes dans le pays, tout nous porte à eroire qu'ils provenaient du Colchicum bulbocodioides et non du Colchicum variegatum. La première espèce, en effet, est la seule qui nous soit connue en Égypte : or, ses tubercules, rarement plus gros que des noisettes et bien souvent plus petits, semblent ne pouvoir être confondus avec les Hermodactes du commerce, bien qu'ils s'en rapprochent par leur surface à peu près lisse et d'un blane jaunâtre sur les exemplaires desséchés ; leur forme est ovale, généralement plus acuminée, el plus aiguë à l'extrémité supérieure. Cette espèce de Colchique, éminemment variable, et qui ne diffère pas assez du Colchicum montanum, L. (1), est très commune dans le nord de l'Afrique, l'Arabie, la Syrie, la Perse, la Mésopotamie, l'Asie Mineure et la Crimée.

ac nos castaneas edimus, multos unica vice, ad impinguescendum, devorant, ex quibus neque alvus aliquo pacto turbatur, neque aliud quippiam mali accidit. Hinc nostri pharmacopoli scire possunt, quantum illis pro vero Hermodactylo utentes, hactenus erraverint. Egroque hos non parum admiratus sum, quando Egyptiæ mulieres earum radicum (quam sine dubio si modo Dioscoridi credendum sit, Colchici esse quisque herbarum materiæ peritus fatebitur) per multos dies ad decem et plures etiam èuntes dormitum sumpsisse instarque castanearum comedisse, sine ulla noxa ibi sæpius compererim. „

sm(1) Voir sur le C. montanum, L., et les espèces analogues, les exetllentes notes de Visiani ( $F l$. dalmatica, I, p. 157 ). desquelles il résulte que l'espèce linnéenne reposant en partie sur une description très incomplète de Loefling , en partie sur deux synonymes faux, on doit rejeter provisoirement le nom de C. montanum et adopter celui de C. Bertolonii, Stev., pour le C. montanum de Desfontaines et de Bertoloni. M. Visiani regarde aussi comme une simple nuance du Colchicum Bertolonii, les exemplaires de Colchique récoltés en Egypte par Figari et qui se rapportent, suivant toute apparance, au C. agyptiacum Boissier.

Du reste, le nom de $C$. Bertolonii, Steven, publié en 1829, dans lẹs Nouveaux 


\section{§ 3. - Hermodacte d'Anguillara.}

Colchicum mostanum, L. (1) (Pro parte, nempe quoad stirpem Lœflingianam hispanicam et exclus, synon. Clusii et Bauhini). Nomen delendum?

Colchicum illyricum, Auct. de mat medic. (Pro parte et excluso synon. Lobel.).

Colchicum montanum, Desf., Fl. Atl.._-Bertol., Rar. ital.pl. Decad.3, p. 19, no 2 et Amøn. ital. p. 24-25 (ann. 1819).

Colchicum Cupani, Gussone, Prodr. Fl. sic., I, p. 452 (ann. 1827).

Colchicum Bertolonii, Steven in Act. nov. Mosq. (ann. 1829). - Visiani, Fl. dalmal. p. 157 (cum annotat. optimis).

Colchicum pusillum, Sieb. exsicc. - Rcem. et Schult. - Kunth.

mém. de la Soc. Imp. des nat. de Moscou, est postérieur de deux ans à celui de C. Cupani, proposé par M. Gussone, dans le Prodromus Fl. Siculce, I, p. 452, ann. 1827 (nous faisons cette citation d'après M. Visiani). Ainsi donc ce serait le nom de $C$. Cupani qui devrait être adopté de préférence à $C$. Bertolonii. Mais s'il était prouvé, comme nous le croyons, que ces deux plantes ne diffèrent pas assez du Colchicum bulbocodioides, M. Biebst., c'est en définitive ce dernier nom qui devrait seul prévaloir.

(1) Les exemplaires suivants de l'herbier Delessert paraissent se rapporter au C. Cupani, Guss. Ils ont tous des feuilles linéaires, au nombre de 2 à 5 , des fleurs (au nombre de 2 à 5 aussi), dont les divisions du périanthe sont linéaires, à 5 ou 6 nervures et dépourvues de crêtes au côté interne.

$1^{\circ}$ Colchicum Cupani, Gussone, récolté à Trapani en Sicile, par M. Parlatore. 1 feuilles, 2 fleurs.

$2^{\circ}$ Colchicum......... Mont Liban. Bové, $\mathrm{n}^{\circ} 513$ (octobre 1832). Trois feuilles linéaires, 2 à 3 fleurs, semblables à celles de l'exempl. précédent.

$3^{\circ}$ Mont Cassius (Palestine). Labillardière; 5 feuilles, 3 fleurs.

$4^{\circ}$ Colchicum monlanum, L. fide Boissier. Sommet de l'Hymette, près d'Athènes; De Heildreich. Automne de 1844. 2 feuilles, 1 à 2 fleurs.

5० Colchicum montanum, Desf,, exemplaires authentiques récoltés par Desfontaines en Barbarie.

Deux de ces exemplaires répondent assez au Colchicum Cupani de Sicile, sauf que leurs feuilles sont un peu plus larges. Un troisième avec des fleurs toutes semblables, mais au nombre de sept, présente deux feuilles beaucoup plus grandes, longues de près d'un décimètre sur au moins un centimètre de largeur. Les divisions du périanthe n'ont pas de crête à la base.

$6^{\circ}$ Colchicum. Alger; champs, mars 1839. Bové in herb Delessert (sous le nom faux de Narcissus Tazzetta). Pareil aux deux premiers exemplaires de Desfontaines.

$7^{\circ}$ Colchicum montanum. Dalmatie; Petter. Feuilles plus larges, bordées de cils denses. 
Colchicum Steveni, Kunth, Enum., IV.

Colchicum Hermoductylum? Parkins., Paradis. (ann. 1629) (1).

Dans beaucoup de livres de matière médicale, on trouve indiqué, comme la source possible de l'Hermodacte, un Colchicum illyricum, que l'on chercherait vainement parmi les espèces de ce genre admises par les botanistes modernes. Linné lui-même, qui cite à tort Miller et Forskähl à l'occasion de ce Colchicum illyricum, ne l'ajamais admis ni défini dans ses ouvrages de botanique. Il s'agit au fond d'une plante tout imaginaire, formée d'éléments hétérogènes, et dont la création est due à deux méprises combinées du vieux de L'Obel. Le célèbre botaniste flamand figura le premier, dans son Stirpium historia (p. 73), une plante qu'il avait reçue, dit-il, d'Alep en Syrie, et dans laquelle il crut reconnaitre à la fois l'Hermodacte des officines, et certain Colchique mentionné par Anguillara (Semplici, p. 275) (2). Il nomma sa plante Colchicum

(1) Parkinson dans son Paradisus, publié en 1629, énumère 19 espèces ou variétés de Colchicum, entre autres la suivante qui est la $13^{\text {me }}$.

13. Colchicum Hermodactylum. Physical Meadow Saffron. - This Physical Meadow Saffron springeth up with his leaves in Autumne, before his flowers appeare beyond the nature of all the former kindes, yet the flower doth, after they are up, shew it selfe in the middle of the green leaves, consisting of six white leaves, with divers chives in the middle, and passeth away without giving any seede that ever I could observe, the greene leaves abide all the winter and spring following, decaying about May, and appeare not until September, when (as I said) the flowers show themselves presently after the leaves are sprung up.

D'après ce qui précède, il est évident qu'il s'agit d'un Colchique à feuilles synanthes, qui peut être le Colchicum bulbocodioides, M. Biebst. Parkinson n'en décrit pas les bulbes. 11 ajoute plus loin, p. 140.

The Colchicum Hermodaclylum may seeme very likely to bee the Colchicum orientale of Mathiolus, or the Colchicum Alexandrinum of Lobelius : and some think it to be the true Hermodactylus, and so call it, but it is not so.

Le Colchicum orientale de Matthiole (Comment. in Diosc. ed.venet., ann. 1565, p. 1108, cum icone) ou Colchicum syriacum Alexandrinum, Lobel (Observ., p. 72, avec vignette copiée de Matthiole, et Advers., p. 54) n'a rien de commun avec les vrais Colchiques. Clusius (Rar. pl. hist., p. 201) suppose que ce pourrait être une Tulipe mal dessinée. Ne serait-ce pas plutôt une Fritillaire?

(2) Il Colchico, che si vede par la Grecia, e nelle parti di Schiavonia nel contorno di Sebenico non è gia quello, che in molti luoghi si trova in Italia : per- 
illyricum sive grceum non venenatum Anguillarce. Peu de temps après, Gerarde (Herbal, p. 160, fig. 6) reproduisit la figure donnée par de L'Obel, en réduisant l'inscription aux seuls mots Colchicum illyricum, forme abrégée, sous laquelle cette prétendue espèce a souvent été reprise par la foule des compilateurs, et même par des auteurs sérieux.

Or voici la vérité sur cette complication de méprises :

1. Le Colchique d'Anguillara n'est pas indiqué comme illyrien, mais comme croissant en Grèce et près de Sebenico, en Dalmatie; donc le nom d'Illyricum n'appartient pas à Anguillara.

$2^{\circ}$ Le Colchique de l'auteur italien est évidemment, ainsi que l'a reconnu M. Robert de Visiani, le Colchicum Cupani, Guss. Bertolonii, Stev.), et n'a pas le moindre rapport avec la plante figurée par de L'Obel.

$3^{\circ}$ Anguillara n'a pas dit, commel'a cru de L'Obel, que son Colchique de Grèce et de Dalmatie fût l'Hermodacte véritable, mais seulement que l'on en substituait les tubercules à ceux de l'Hermodacte (Le sue radice si cavano et si portano a vendere per la Italia in vece di Hermodattili).

$4^{\circ}$ Entin la figure de ce prétendu Colchicum illyricum, telle qu'elle est donnée par de L'Obel, non-seulement ne représente pas un Colchique, mais ne rappelle même aucune plante connue. On y voit un bulbe semblable à celui d'un Narcisse, une tige avec des feuilles distiques nerveuses, qui rappellent celles des Epipactis, des fleurs à six pièces en étoile, qui semblent se rapprocher de celles des Gagea, d'autant plus que de L'Obel les dit jaunatres; bref, I'ensemble présente quelque chose de discordant avec toutes les formes connues de Monocotylédones. On peut supposer, sans trop d'injustice pour la mémoire de l'auteur flamand, qu'il a, dans cette circonstanee, construit innocemment un assemblage de pièces disparates : Bonus quandoque dormitat Homerus.

eioche esso fa le foglie strette, simili al Butho, storte per terra, et le sua radici si cavano et si portano a vendere per la Italia in vece di Hermodattili. Produce all' Automno i fiori simili a quelti del Croco, ma molte minore, tre e quattro in cima di un fusto alto una spanna. Nel rimanenta Dioscoride si legga, il quale ha à pieno sodisfatto. 


\section{§ 4. - Hermodacte de Mathiole.}

Hermodactrues tuberosus, Salisb. in Transact. of the Hort. Soc., I, p. 304 (nomen tantum). - Parlatore, nuove gen. e nuove sp. di piante monocot. (ann. 1854), p. 45. - Godron in Gren. et Godr., Fl. franc., III, p. 245 (ann. 1855).

Iris tuberosa, Dodon, Pempt., p. 249 (cum icone).-De L'Obel. Observ. 51 (cum icone præced.). - Parkins., Parad., p. 188, tab. 185, f. 6. - L, Sp. p. 58.- Willd., Sp., I, p. 240. - Vahl, Enum., II, p. 152. - Rœm. et Schult, Syst., I, p. 480. — Bot. mag., t. 531. - Redouté, Lil., t. 48. Sibth. et Sm., Fl. grace, I, p. 25, t, 41 .

Hermodactylus verus, Matth., Comment,, edit. 1565 , p. 1109 (cum icone). - Camer. De pl. epit., etc. 847.

Iris uberosa Belgarum et secundum Aldroandum prima Lonchitis Dioscoridis, de L'Obel, Icon. 98 (ann. 1581). - J. auh., Hist. plant., p. 730 (cum iconea Lobelio mutuata).

Iris tuberosa flore obscure viridi colore, Sweert, Florileg, tab. 36, fig. dextra (ann. 1612 et 1620 ).

Iris tuberosa folio anguloso, C. Bauh., Pinax, p. 40.

Iris tuberosa folio anguloso flore obscure viridi colore, Moris.. Hist., vol. I, pars 2, p. 348 , sect. IV, tab. 6 (ann. 1745).

Hermodactylus folio quadrangulo, Tournef., Coroll. 50. - Miller, Dict., édit. 8 (2 vol. in-fol., 1768), voce Hermodactylo.

Persuadé que l'Hermodactyle des Arabes et l'Hermodacte des officines n'étaient pas les mêmes què l'Hermodactylos des Grees, André Matthiole s'efforesa de retrouver ce dernier en dehors des espèces de Colchique. Il songea d'abord au tubercule palmé de l'Orchis maculata, qu'on appelait alors Palma-Christi, et dont la forme aurait pu justifier à quelques égards le nom d'Hermodactylos. C'est l'opinion qu'il présente, avee réserve, dans la belle édition latine de ses Commentaires, publiée chez Valgrisi, à Venise, en 1554. Mais, plus tard, dans l'édition de 1565, il eroit fermement avoir retrouvé le véritable Hermodactylos des Grecs dans une plante que lui avait envoyée de Constantinople le célèbre Augier de Busbeke, ambassadeur de I'Empire auprès du sultan, le même à qui l'Europe occidentale doit l'introduction duLilas et du Marronnier d'Inde. Cette plante, que Matthiole a grossièrement figurée sous le nom d'Hermodactylus verus, n'est rien autre que l'Iris tuberosa de Linné ou l'Hermodactylus tuberosus de Salisbury. 
L'auteur paradoxal de la Physiognomonie, Baptiste Porta, n'a pas oublié l'Hermodactyle parmi les plantes signaturées, c'est-à-dire dont certains organes, répondant par quelque vague ressemblance à des parties du corps humain, doivent servir à la guérison des maux dont ces parties sont affectées. D'après ce système, la racine d'Hermodactyle ne pouvait manquer de rappeler la forme d'une main d'homme. Aussi Porta, suivant l'opinion de Matthiole, figure-t-il comme Hermodactyle l'Iris tuberosa, auquel il donne une racine à cinq branches disposées comme les doigts d'une main, sans oublier même des indices d'ongles (voir Porta, Phytognomonica, p. 140 ; Napoli, in-4). L’Hermodactyle est naturellement rapproché, dans ce chapitre, de l'Orchis maculata, vulgairement appelé Palma-Christi, et du Digitaria sanguinalis ou Gramen digitatum.

En 1703, Tournefort, dans son Corollarium, crut devoir faire un genre spéeial de l'Iris tuberosa, genre pour lequel il adopta lè nom d'Hermodactylus emprunté à l'ouvrage de Matthiole. Il semblait par cela même adopter l'idée du commentateur de Dioscoride à l'égard de l'identité supposée entre cet Iris tuberosa et l'Hermodactylos des Grees. Mais, en ce qui regarde les Hermodactes des officines, ni Tournefort, ni Matthiole, $n$ y voyaient des tubercules de Colchique.

Linné le premier, interprétant à faux les opinions de Matthiole et de Tournefort, regarde l'Hermodacte officinal comme produit par l'Iris tuberosa, auquel il rapporte en conséquence comme synonyme le Colchicum radice siccata alba de Gaspard Bauhin (Linn., Mat. met., edit. Amsteldami, 1749, p. 9). Il constate pourtant que beaucoup d'auteurs rapportent l'Hermodacte au Colchicum foliis undulatis patentibus, Hort. Cliff., c'est-à-dire au Colchicum variegatum.

Plus tard, en 1772 (1), il ajoute une nouvelle erreur à la première, en attribuant à Miller et à Forskähl l'idée que l'Hermodacte provient du Colchicum illyricum. Nous n'avons pu trouver une

(1) Diss. Observ. in mat. med., 1772, p. 6, cité par Murray. Voir aussi p. 48. 
telle mention chez ces auteurs, et P'on a vu ce qu'il faut penser du prétendu Colchicum illyricum.

Égarés par l'auterité si puissante de Linné, divers auteurs, même récents, ont cité l'Iris tuberosa comme la source de l'Hermodacte. Mentionnons dans ce nombre Desbois de Rochefort (1), Sprengel (2), Mérat et de Lens (3), Fraas (4) et M. de Martius (5).

Mathias de L'Obel (Observat., p. 51) cite vaguement Ulysse Aldrovande et autres auteurs italiens, à l'appui de l'idée que le Lonchitis prior ou prima de Dioscoride (6) serait identique avec l'Iris tuberosa. Cette idée, adoptée sans discussion par Sibthorp et Smith (Flora greca), nous parait bien loin de pouvoir se passer

(1) Cours de mat. med., I, p. 375.

(2) Historia rei herb., I, p. 218.

(3) Dict. de mat. med., articles Hermodacte et Colchique.

(4) Synops. Fl. class., p. 293 (München, 1845).

(5) Pharmakngnosie (citation de Pereira), et Syllab. Prcelect. de bot. pharmac,med., p. 8 (sans date).

(6) Lonchitis prima Discoridis. - Lonchitrs folia habet porro perquam similia, latiora tamen et rubentia, plurima ad radicem circumfracta, veluti in terra procumbentia. Habet et circa caulem pauca, in quo quidem flores ceu pileoli, hiantibus comicis personis figura miles, iique nigri, sed e rictu albam veluti parvam linguam exserentes, quæ ad labrum inferius spectet; semen involucris clausum, triangulum et lanceæ cuspidi simile, unde etiam planta ipsa sibi cognomentum arrogavit; radix dauci. Nascitur in asperis ac sitientibus. Radix ex vino pota, urinam ciet.

Dioscorides lib. III, cap. CLXI (ed. Sarracenus). Pas de note de Sarracenus sur ce chapitre.

Le Lonchitis altera du chapitre suivant est une fougère.

Le texte précédent est transcrit de la traduction de Dioscoride de Johannes Rivius (p. 281), où l'on trouve annexée la note suivante de Gualterius Rivius :

Lonchitis Dioscoridi duplex est. Prima habet folia porri, ut Dioscorides trädit, rubentia ad radicem et plura quam in caule, capitula personis comicis similia, parvam exserentibus linguam, radicibus prælongis. Nascitur in sitientibus, sæpe in aridis et petrosis invenitur; sed nullum succurrit nomen dignum, quo inscribatur. Pueri qui montibus aberrant, nigros capettos, quasi Galeros, germanice Schwartz Kappen, nominant. Altera Lonchitis, etc.

Ce qui suit se rupporte à la fougère représentée sur la vignette, et qui est le Blechnum Spicant.

$\alpha$ Lonchitidis quæ semen triangulare habet, velut cuspis, radix similis dauci 
dé preuves, et le problème reste soumis à la sagacité des botanistes futurs.

Il serait superflu de prouver que l'Hermodacte officinal ne peut avoir rien de commun avec l'Iris tuberosa. On ne saurait alléguer aucune raison solide en faveur de lidentité de ce dernier et de l'Hermodactylos dès Grees. L'Hermodactylos provoquaif des nausées; ainsi fait le Colchique : les tubercules d'Iris tuberosa, d'abord assez agréables au goùt, laissent bientôt dans la bouche une impression âcre qui dure des heures entières, mais qui n'a rien de nauséeux. Nous en avons fait l'expérience au moyen d'un demitubercule. Une dose plus forte produirait peut-être de fầcheux effets.

Tournefort, on l'a vu, distingua le premier génériquement l'Iris tuberosa sous le nom d'Hermodactylus; mais il n'en signala pas le caractère le plus important, l'existence d'un ovaire uniloculaire constatée en premier lieu par notre ami M. Parlatore.

\section{$\S 5$. - Hermodacte de Brunfels.}

Otho Brunfels, dans son ouvrage intitulé : Herbarum vivae eicones (ann. 1532), confond l'Hermodactyle avec le Narcissus, et représente comme tel (page 129 du tome Ier) deux plantès bien différentés, le Narcissus Pseudo-Narcissus etle Galanthus nivalis.

§6. - Pseudohermodactylus de Mathiole.

Erythronium Dens canis, L.

Sous ce nom de Pseudo-hermodactylus ou faux Hermodacte, radici urinam ciet; ejus vero quæ Scolopendrio similis est folia viridia quidem vulnera ghitinant, sicca autem cum aceto pota, lienes indurates sanant. n

Paulus Egineta, De re medica, lib. VIF, p. 631. G., édit. Henri Estienne, dans la collection des Medica artis principes (ann. 1567).

« Lonchitidis triangulare semen hastæ simile habentis, radix urinam ciet; ejus vero quæ Scolopendrio similis est folia viridia vulneribus glutinandis conducunt. Sicca eadem ex aceto pota, lienes induratos sanant. »

Aetius, Tetrabiblos, sermo 1, p. 41 A, édit. Henri Estienne, dans la collection 
Matthiole a figuré dans ses Commentaires (1) une plante que l'on prenait souvent, a cette époque, pour l'Hermodacte (2). Il est difficile, au premier abord, de reconnaitre dans cette informe vignette un type quelconque de la flore d'Europe. Ce n'est rien autre pourtant que l'Erythronium Dens canis, ainsi que I'a reconnu de L'Obel (Observ., p. 97), et que l'ont admis Gaspard Bauhin (Pinax, p. 87) et Dalechamp (Histor., p. 1583).

\section{\$7. - Hermodacte de quelques auteurs allemands.}

Cyclamen europæum, $\mathbf{I}$.

Jérôme Tragus (3), confondant, ainsi que nous l'avons dit plus haut, l'Hermodacte des officines avee le Colchique d'automne, et recommandant contre la gontte l'usage externe de ce dernier tubereule, signale, en outre, comme étant l'Hermodactyle de quelques aufeurs, un Cyclamen, qu’il déeril et figure plus loin, et dans lequel il est aisé de reconnaìtre notre Cyclamen europceum.

(4) A la page 1140 de l'édition latine publiée à Venise, en 1565 , et p. 7\%, de l'édition des ceuvres complètes (Opera omnia, etc), publiée en 1598, par Gaspard Bauhin. Cette dernière figure, bien qu'assez grossière, est pourtant bien meilleure que celle publiée par Matthiole.

(2) Conrad Gesner, citẻ par G. Bauhin (Pinax, p. 87), a considéré THermodactyle de Mésué comme étant la plante aujourd'hui nommée Erythronium Dens canis

Voici le synonyme, tel que le cite G. Bauhin :

Hermodactylus Mesuæi, folio maculoso et Dens canis Gesn. Hort.

- Woiei la citation complète de C. Gesner :

s Hermodactylus Mesuæ folio maculoso, K. (c'est-a-dire Joachimns Kreichius, Torgensis pharmacopola). Cirea Bononiam ubi sponte abundat Dentem canis vocant; aliqui non recte pro Satyrio Erythraico ostendunt aut hanc aut similem quandam. ")

(C. Grswer, Hort. germ., p. 261 (ouvrage publié avee diverses cuvres de Valerius Cordus, en 1564 ).

Lemème Conrad Gesner, dans l'Herbarum nomenelatura, imprimé à la suite de la traduction de Dioscoride par Jean Rivius, $p$. 547 , cite 1 Hermodactylos - comme synonyme du Quinquefolium, qui doit être, suivant toute apparence, te Potentilla Tormentilla. Mais on ne saurait assurer, sur ce simple mot, que l'illustre zateur eût en vue l'Hernodactylos d'Alexandre de Tralles.

(3) De stirp. Comment. interprete D. Kybero, lib.'II, eap. cxxvi. 


\section{$\S 8$. - Hermodactylos de Myrepsus et d'Actuarius.}

Myrepsus (Nicolaus-Alexandrinus), dans le premier chapitre de son livre De compositione medicamentorum (collection des Medica artis principes de Henri Estienne, ann. 1567), énumère, parmi les nombreux ingrédients de ce qu'il appelle Antidotus aurea Alexandri, l'Hermodactyle rouge et blanc, au sujet desquels son annotateur, Leonhardt Fuchs, fait l'observation suivante (p. 355, B.) :

" Per Hermodactylos hoc loco et alias etiam Nicolaus intelligit radices, quas officinæ hodie vocant Behen albam et rubeam. Quod etiam facit Actuarius (1) ut copiosus lib. I de compositione medicamentorum ostendimus. Et monendus hic nobislector erit, nonnulla hoc loco in latinis desiderari codicibus, quæ quisque facile ex collatione cum nostra conversione deprehendet (2). »

(1) Autres passages de Myrepsus, relatifs à l'Hermodactylus. - a Antidotus ex hermodactylo admodum bona. Recipit hermodactyli unciam unam semis, anisi, cumini æthiopici, hoc est carnabadii, ameos, epithymi, piperis communis, zingineris singulorum scrupulum semis, thymi hexagium unum, alipiæ unciam unam. Datur cum melicrato. " (Myreps. De Antidotis, sect. I, cap. ccccxxix.)

a Antidotus alia purgans ex hermodactylo, conferens omnibus malis defluxione natis, podagricis, arthriticis et malo corporis habitu correptis. Habet cinnamomi unciam semis, croci hexagium unum, zingiberis unciam semis, etc., etc., suivent d'autres substances très nombreuses, hermodactyli drachmam unam... sacchari et stillatitii rosarum quod satis est: „ (Ibid., cap. Cccclxxvm.)

a Antidotus ex hermodactylo, conferens ad podagricos, arthriticos et quosvis dolores, omne denique vulnus et ad omnes actiones. Accipit hermodactyli hexag. unum semis, cumini æthiopici, colcynthidis, unc. quinque; chamædryos unc duas et quartam partem, centaurei, etc., etc. Suivent d'autres substances très diverses et incompatibles. ' (Ibid., cap. CcccLxur.)

a Antidotus ex hermodactylo auxiliatur podagræ, a frigido humore ortæ. Habet decocti radicum foeniculi lib. duas, mellis despumati libram unam. Ebulliant and mellis crassitudinem : postea injicito hermodactyli et turpethi puri singulorum unciam unam. Confecta, usu postulante, datur nucis avellanæ quantitate jejuno et vino. n (Ibid., cap. cxxv.)

Suit une autre recette plus complexe.

(2) " Ex hermodactylo (sous-entendu Antidotum) podagricis et arthriticis, ut quos succos crudos ex alto detrahit, prodest: hermodactyli, galangæ, pyrethri, 
L'idée exprimée dans le passage de Fuchs sur l'identité de l'Hermodactyle de Myrepsus et d'Actuarius avec le Behen blane et rouge, est également celle de Matthiole. Seulement Fuchs parle du Behen alba et rubra des officines de son temps, tandis que, d'après Matthiole (page 536 de l'édition latine de ses Commentaires, publiée à Venise en 1554), il s'agirait du Behen des Arabes qui n'était pas celui des officines d'Italie, mais bien une racine tortueuse odorante, semblable à celle du Pastinaca. Enfin, Jean Bodæus a Stapel (1) rapporte l'Hermodactylos de Myrepsus et d'Actuarius au Behen de de Sérapion, dont il existerait, dit-il, deux espèces : l'une rouge (rubra), l'autre blanche (alba), toutes deux constituant des racines analogues à celles du Pastinaca, odorantes, tortueuses, originaires d'Arménie. Ce commentateur cite une phrase de Myrepsus (Antidot. 38), dont il dit que le texte est altéré, et qu'il corrige de façon à lui faire signifier en latin : Behen alba et rubrce qua nimirum Hermodactyli longi dicuntur.

Nous rapportons ces opinions pour ce qu'elles valent, dans tout leur vague, avec leurs contradictions, en avouant notre impuissance complète de les juger, faute surtout de renseignements positifs dans les textes qui font l'objet de la question (2). Observons seulement qu'il faut se garder de confondre le Behen de Sérapion, de Myrepsus et d'Actuarius, avec le Ben de Mésué, e'est-à-dire la Noix de Ben du commerce, glans unguentaria des Latins, qui est la graine du Moringa aptera, Gærtn.

\section{§9. - Hermodacte de Finch.}

Trapa natans.

La graine farineuse du Trapa natans ou Châtaigne d'eau, dépouillée de ses téguments, ressemble assez au tubercule de

zingiberis, zaduaris, anisi, gentianæ, aristolochiæ, singulorum quadrans, mellis sufficiens modus. \#

Actuarius, œuvres, livre V, p. 264 B. dans la collection des Medicee artis principes, de Henri Estienne, 1567

(1) In Theophrasti Hist. plant., Amsteld., 1544 , p. 299.

(2) Consultez là-dessus de l'Obel, Stirp advers. nov., p. 55. 
l'Hermodacte, pour que des personnes étrangères à la botanique aient pu confondre deux choses au fond si différentes. Ainsi paraît avoir fait un certain marchand de Londres, nommé Finch, dont l'opinion sur ce point est rapportée dans le Theatrum botanicum de Parkinson (p. 1587). On peut rapprocher de cette opinion celle de Pomet (1), qui, sur la foi de certains correspondants marseillais, donne gravement les Hermodactes pour le fruit d'un arbre d'Egypte. Enfin, et pour clore cette longue liste de méprises, èitons Virey (2) qui, rapportant l'Hermodacte au Colehicum illyricum, ajoute qu'on l'attribue également à la racine (sic) de l'A maryllis lutea.

\section{DEUXIÈME PARTIE.}

DES ORGANES VÉGÉTATIFS HYPOGÉs DES COLGHICUM ET DE L'HERMODAGTYLUS TUBERosUs (Iris tuberosa).

\section{§1. - Tubercule des Colchicum.}

Dans la première partie de ce Mémoire, nous avons employé sans explication le mot tubercule, pour désiguer ce qu'on appelle souvent bulbe, ou même racine de Colchique ef d'Hermodacte. C'est qu'en effet, ces corps tubéreux, tels qu'on les emploie en médecine, n'ont rien de commun avec les vrais bulbes, et moins encore avee des racines. Tächons d'en préciser netlement ici la nature morphologique et les rapports avec des organes analogues.

Pris dans son ensemble, le soi-disant bulbe de Colchique tient à la fois du bulbe par ses tuniques externes et du tubercule par son contenu solide. Il mérite done le nom de bulbo-tuber que Gawler a proposé, et que nous voyons adopté dans la Théorie élémentaire de De Candolle ( $3^{\mathrm{e}}$ édition, p. 305). Le mot cormus, employé dans ce sens par beaucoup de botanistes anglais, a reeu des aceeptions très diverses, et n'est pas généralement admis dans la séience: celui de bulbe solide a le défaut de laisser confondus des organes très distinets, tèls que les bulbes de Tulipes presque entièrement composés de feuilles squamiformes, charnues, et les faux bulbes

(1) Histoire des drogues, in-4, 1694, p. 210.

(2) Histoire nquirelle des médicaments, Paris, 4820. 
des Crocus et des Colchiques, dont là masse interne est formée de rentlements de l'axe caulinaire. En somme, c'est done le terme bulbo-tuber que nous adopterions volontiers dans une description latine; mais, conme ce mot traduit sonnerait mal à des oreilles françaises, nous emploierons, faute de mieux, le mot tubercule tout court, en sons-entendant « revêtu de tuniques. »

La structure générale, la végétation et la signification morphologique du tubercule du Colehique, sont parfaitement coneus et merveilleusement exposés dans le Genera plantarum d'A.-L. de Jussieu (1). Tous les faits essentiels du sujet, condensés dans cette note descriptive, furent développés avec soin dans un travail spécial de M. Jules de Tristan (in Mém. du Mus., t. X, p. 36-57, tab. 1-3). Ge serait done chose superflue d'y revenir en détail. Rappelons-en juste assez pour l'intelligence des observations auxquelles cette structure donne lieu.

A l'époque de la floraison, le tubercule du Colchicum autumnale est enveloppé de deux tuniques parfaitement closes(2), dont le sommet se prolonge en gaîne autour de la tige florifère. Ces tuniques représentent des feuilles imparfaites ou, si l'on veut, des spathes, développées l'automne précédent à la base de la tige alors flori-

(1) «Ex persistente pristini caulis tubere striato dilatato hine sinuato et tunicis foliorum radicalium præcedentium vestito, lateralis infra exoritur sub iisdem tunicis nova plantula basí tuberosa et subtus bulborum more radicans, in tuberis pristini semiamplectentis sinu recepta. Huic spatha exterior radicalis cylindrica tubulosa, apice hinc fissa semi subterranea; flores $2-6$ ex spatha semi-prodeuntes absque foliis; fructus interdum flore multò tardiores, assurgenti extra spatham cauli insidentes; folia cum fructibus eminentia nervosa, radicalia vaginantia, caulina semi-amplexicaulia. Increscens planta suum expandit tuber, pristinum hine exsugit opprimitque mox periturum, novamque simul inde basi trudit sequentis plantulæ gemmam. Priscum tuber tum habitu tum et usu quasi assimilandúm perispermo aut et cotyledoni, utrique in monocotyledonibus laterali proprio succo plantulam enutrienti, et ipsa adultiore marcescenti ac evanido. Duplex plerumque simul ex eodem tubere exilit gemma lateralis, altera hinc inferior jam descripta et frugifera, altera inde superior pariter caulescens sed gracilior et vix florifera. „ Juss., Gen., p. 47 (ann. 1789).

(2) Il n'y a souvent qu'une tunique : ce cas est fréquent chez le $C$. variegutum. Quelquefois la gaîne intérieure se prolonge en limbe foliacé; une des feuilles s'insère donc alors à la base meme du tubercule. 
fère, tige dont il ne reste d'autre trace que sa base renflée en tubercule charnu. Ce tubercule, lentement formé d'un automne à l'autre, porte actuellement deux gemmes : l'une en partie développée, puisqu'elle est en train de fleurir; l'autre, moins avancée, souvent avortée ou du moins peu développée, et comme supplémentaire. La première gemme, ou mieux la tige fleurie, s'insère à la face antérieure du tubercule, sur un processus unguiforme de sa base; elle se loge en partie dans une gouttière, que sa pression a produite sur la face du tabereule. De sa base naissent de nombreuses fibres radicales, embrassées par une courte coléorhize à deux lèvres transversalement étendues. Sa première feuille est une gaine scarieuse dont il ne reste que des lambeaux à l'époque de la floraison, et qui nous a paru opposée à la gaine qui lui est immédiatement superposée. Les deux suivantes sont des gaînes encore cylindriques et blanches, enveloppant les fleurs et les jeunes feuilles, gaines destinées à revêtir l'année suivante le nouveau tubercule que va former, en se renflant, la base de la tige actuellement fleurie. La seconde gemme est logée dans un sillon, sur la convexité dorsale et près du sommet du tubercule; elle pousse également de sa base un faisceau de fibres radicales, embrassées par une coléorhize à lèvres étroites. Au dessus de ce bourgeon dorsal, et sur la pointe même du tubercule, on voit les restes desséchés de la tige dont le tubercule formait la base, tige qui s'est détruite après avoir mûri ses fruits. Enfin, vers la base du côté convexe ou dorsal du tubercule, se montre una cicatrice arrondie, trace de son insertion sur l'ancien tubercule dont il dérive.

En somme, le tubercule du Colchique, abstraction faite de ses tuniques, représente une base de rameau portant deux bourgeons, dont un antérieur ou ventral, l'autre postérieur ou dorsal; le nombre de ses mérithalles est de trois, savoir : un entre la base du bourgeon antérieur et la base même du tubercule, un autre entre le sommet du tubercule et le bourgeon postérieur, le troisième enfin entre les deux bourgeons.

La position de ces bourgeons sur le tubercule semble les rendre propres à équilibrer le développement de la plante de telle sorte 
qu'elle ne s'enfonce pas trop bas dans le sol, ou ne s'élève pas trop près de la surface. La gemme antérieure, en effet, naissant sur le processus inférieur du tubercule, tendrait toujours à porter plus bas les tubercules successifs; mais cet effet se contre-balance apparemment par le développement occasionnel de la gemme supérieure.

Les différences si remarquables que nous avons signalées chez les tubercules du Colchicum variegatum se retrouvent probablement chez d'autres espèces. On peut présumer que la forme arrondie provient de la gemme inférieure, et la forme dactyloïde, plus maigre, de la gemme supérieure (1). Le processus inférieur du tubercule de Colchique acquiert parfois un développement très considérable par rapport au corps même du tubercule. Nous en avons un exemple très remarquable sur un pied de Colchicum Balansce, chez lequel ce processus présente la forme et les dimensions d'un doigt index de grosseur moyenne. On conȩoit parfaitement que des tubereules semblables aient pu suggérer l'idée du mot Hermodactylos. M. Balansa pense, avec toute apparence de raison, que ces bulbes à processus très longs se trouvent trop près de la surface du sol, et tendent à s'y enterrer plus avant.

Il existe entre le tubercule de Colchique muni de sa gemme florifère, et l'embryon de eertaines Monocotylédones, particulièrement des Graminées, des ressemblances on ne saurait plus frappantes. Antoine-Laurent de Jussieu s'est contenté de les rappeler avec sa prudence habituelle, sans en induire autre chose qu'une certaine analogie physiologique. M. Jules de Tristan, partageant cette réserve, fait pourtant un pas de plus dans cette assimilation, dont il établit en détail les termes équivalents. Ainsi, suivant la terminologie de L.-C. Richard sur les embryons, le corps du tubercule répondrait à l'hypoblaste des Graminées et la gemme antérieure au blaste. Ce rapprochement, plus ingénieux que juste, ne supporte pas un long examen. L'hypoblaste, en effet, dont L.-C. Richard fait une radicule, Mirbel un cotylédon, Adrien de Jussieu un appen-

(1) C'est peut-être au développement successif de plusieurs générations de ce bourgeon rachitique, qu'est due la diminution de vigueur et de volume signalée par Parkins, he le Colchicum variegatum des jardins de l'Angleterre. 
dice de la tigelle, et M. Germain de Saint-Pierre un cotylédon dans le haut, une radicule dans le bas, l'hypoblaste ne porte pas de bourgeons dorsaux. Le tubercule du Colchique représente, au contraire, un axe à plusieurs mérithalles, à deux bourgeons, dont un dorsal : la gouttière, dans laquelle se loge en partie le bourgeon antérieur, ne se forme qu'à mesure du développement de ce bourgeon en tige fleurie. On ne saurait non plus établir un juste rapprochement entre le tubercule du Colchique et les embryons dits. macropodes des Zostéracées, si bien étudiés par Adrien de Jussieu (Ann. des sc. nat., 2 ${ }^{\text {e }}$ sér., t. XI, p. 35Ц-6, tab. XVII, fig. 15 et 16). Les véritables rapports des tubercules-tuniqués des Colchiques sont, ainsi qu'on l'a depuis longtemps pu reconnaître, avec les prétendus bulbes des Crocus et d'autres Iridées (Ixia, Sparaxis, ete.), avec certains pseudobulbes d'Orchidées (Cologyne, Laelia, etc.); mais il y a là des différences de détail que nous omettons à dessein en ce moment.

\section{§2.-Tubercules de l'Hermodactylus tuberosus, Salisb. (Iris tuberosa).}

Jusqu'à ces derniers temps, on a mentionné comme des racines les tubercules de l'Hermodactylus túberosus. Tout récemment, M. Parlatore en les décrivant (1) ne s'est pas expliqué leur nature, probablement parce qu'il les a vus trop jeunes; et M. Godron (2), qui seul les indique avec raison comme des rhizomes, ajoute, à tort, que ces rhizomes sont nus, probablement aussi faute de les avoir vus sous divers états (3). Afin de mieux saisir cette singulière

(1) "Questa specie manda in basso due o tre tuberi alungati, quasi cylindrici e ottusi all'apice, di colore bianchiccio, dalla base di ciascuno dei quali parte di sopra una foglia, coperta in basso da guaine proprie e da altre guaine comuni anche al fusto che sta nel mezzo e che porta il fiore: dalla base medesima ma di sotto partono delle fibre radicali bianche et lisce. ' (Parlatore, Nuovi gen. $\boldsymbol{e}$ n. sp. di piante monocot. Firenze, in-8, 185 i, p. 47.)

(2) Sonche formée de plusieurs tubercules nus et oblongs et de fibres radiculaires, Godr, in Fl. franc,, vol. III, part. I, 1855

(3) M. Germain de Saint-Pierre, possédant depuis quelques années des notes et des dessins sur la structure de ces rhizomes, en a fait l'objet d'une communication à la Société botanique de France, immédiatement après l'exposé de nos propres observations. (Séance du 28 décembre 1855.) Nos opinions diffèrent sur.

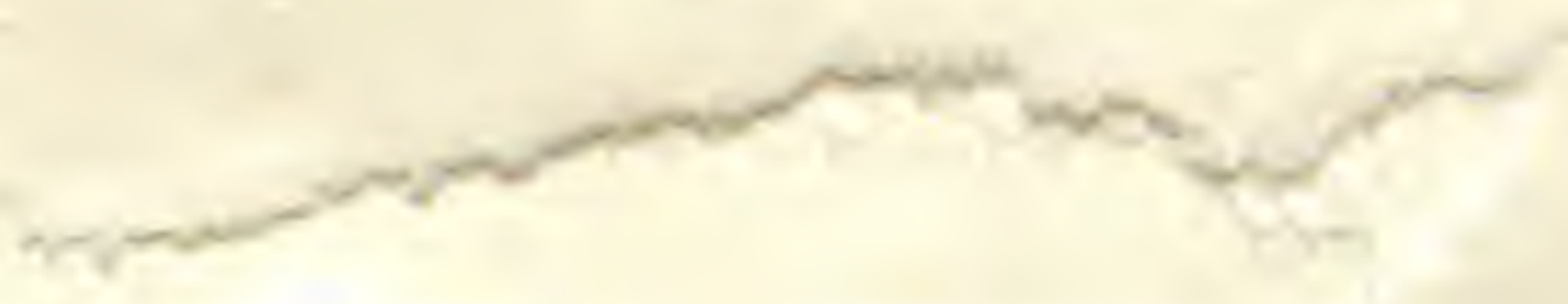


organisation, nous étudierons l'Hermodactylus à deux périodes de végétation bien différentes, savoir : au printemps, lors de l'anthèse, et vers les premiers jours de décembre, quand ses liges aériennes ont disparu.

Un pied d'Hermodactylus tuberosus, pris à l'époque de la floraison, présente à sa base les parties suivantes (fig. 5): En $r$, une souche brune, ligneuse, dépourvue de fibrilles radieales, sauf au point où s'insèrent les squames ou gaines scarieuses, qui sont les premières feuilles de la tige principale $u$. Cette tige fait suite au rhizome, par rapport auquel elle est terminale; elle émet de sa base même deux ou rarement plusieurs tubereules, qui naissent chacun à l'aisselle d'une gaine, et la déchirent pour s'étendre horizontalement au dehors. Chaque tubercule en particulier s'insère sur la tige primaire par un pédicule extrêmement courl, où, pour mieux dire, il est presque sessile. Près de sa base, du còté supérieur, il semble émettre un bourgeon feuillé, comprenant une gaine cylindrique et scarieuse $v$, autour d'une seule feuille verte; mais cette gaîne et cetle feuille, au lieu de naître du côté supérieur du tubercule, comme a cru le voir M. Parlatore, s'insèrent circulairement autour du tubercule lui-même, qu'elles enveloppent d'abord en entier, et qui les déchire l'une après l'autre, comme il a d'abord déchiré la gaine $t$. Dans l'état que représente la figare 5 , la base de la gaine $v$ est déjà déchirée par le tubercule, et l'on en voit en $y$ un des lambeaux. Quant ì la base de la feuille verte, fortement distendue en coecum par le développement du tubercule, elle forme encore autour de cet organe un sac membraneux, sur lequel se dessinent, comme les côtes d'une cage thoracique de Vertébré, des nervures courbes convergeant vers la ligne médiane supé-

les points suivants : $4^{\circ}$ M. Germain n'a pas représenté comme nous dans ses dessins linsertion des feuilles dont les bases renflées servent de tunique au tubercule; $2^{\circ}$ il a cru voir soudées en une seule les deux tuniques, selon nous dis-

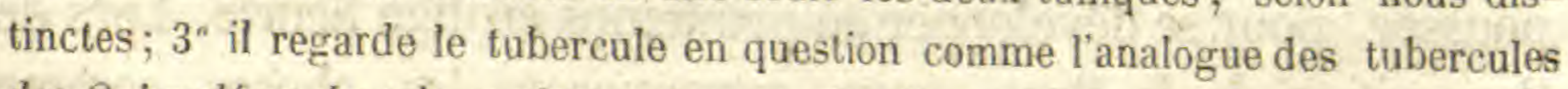
des Ophryđlées, dont la surface même serait, d'après lui, formée par une feuille enveloppant une masse radiculaire simple ou multiple. Ces divergences sur des points de fait et d'interprétation seront mises en rogard dans le. Bulletin de la Société botanique. Il suffit ici de les avoir sommairement signalées. 
rieure, et surtout vers le point où le limbe de la feuille semble sortir du tubercule. Le tubercule lui-même, dépouillé de ses deux téguments, se présente comme un corps oblong, lisse, encore dépourvu de fibres radicales et de bourgeon apparent.

Examinons maintenant ces tubercules, tels que nous les avons reçus de M. Van Houtte dans les premiers jours de décembre, détachés du rhizome qui leur a donné naissance. La figure $b$ en représente deux accolés ensemble par la base. Ils sont cylindriques oblongs, un peu recourbés à leur bout, que termine un bourgeon formé d'écailles blanches et de feuilles encore rudimentaires : en dessous, au point où l'extrémité se recourbe vers le haut, s'insèrent en demi-cercle des fibres radiculaires. Sur la ligne médiane inférieure, on voit, en $m$ et $n$ (fig. 7), deux espèces de taches ou chalazes, dont le disque représente une portion nue du tubercule, et dont le pourtour marque une portion de la ligne d'insertion de feuilles dont on ne retrouve plus que des restes. Ces restes, sous forme d'une pellicule et d'un réseau fibreux étroitement appliqués à la surface du tubercule, appartiennent évidemment aux bases de la gaine et de la feuille qui formaient au printemps les tuniques du tubercule.

D'après ce qui précède, le tubercule se dévoile comme un vrai rhizome, naissant sur la tige principale, à l'aisselle d'une gaîne dont il déchire la base, phénomène très fréquent, on le sait, chez les Monocotylédones, par exemple, chez nos Orchidées tubéreuses, chezles Cypéracées, ainsi que chez le $\mathbf{N}$ elumbium. Ce rhizome tubéreux présente deux phases principales d'évolution : l'une au printemps, alorsque son axe reste enveloppé par les bases distendues et sacciformes de deux feuilles, dont l'une est réduite à l'état de gaîne scarieuse, et l'autre est munie d'un limbe linéaire vert; la seconde phase est automnale, alors que la gaine et la première feuille ont disparu, et que le bourgeon terminal du tubercule commence à pousser, pour se développer au printemps en tige fleurie. Lors de l'apparition de la fleur, nous retrouverons les choses comme dans la figure 5, c'est-à-dire que le tubercule sera devenu souche ligneuse, et que de nouveaux tubercules seront en voie de formation. 
AU POINT DE ve Botanique et PHarmaceutiQue.

Pour se figurer comment les deux feuilles ( gaîne et feuille parfaite) enveloppent d'abord le tubercule ou l'axe charnu dont elles dérivent, on peut les comparer aux deux téguments d'un ovule campylotrope dont la base et le sommet se touchent. Nous supposons que la base des deux premières feuilles du tubercule est à leur ligne d'insertion réelle, et leur sommet au point où elles ont l'air de s'insérer sur le tubercule.

Observons que le côté dorsal, ou la ligne médiane postérieure des deux bases distendues des feuilles, est celui sur lequel elles sont déchirées par le tubercule.

Il n'existe au fond aucune différence essentielle entre le tubercule de l'Hermodactylus tuberosus et les branches du rhizome rampant de beaucoup d'Iris, tels que l'Iris germanica. Seulement ces derniers rameaux ne sont pas revêtus et complétement cachés, dès l'abord, par des bases de feuilles renflées en coecum, lesquelles ne livrent passage que par une déchirure à l'extrémité de l'axe dont elles dérivent. Les diversités sont bien plus grandes entre les tubercules de l'Hermodactylus et les ognons de l'Iris Xyphium, qui sont de véritables bulbes écailleux.

\section{Conclúsions géNÉrales.}

$1^{\circ}$ L'Hermodactylos des médecins grecs est génériquement identique avec l'Hermodactylos ou Surugen des Arabes, et avee notre Hermodacte officinal.

2. L'Hermodacte officinal provient, suivant toute probabilité, du Colchicum variegatum.

$3^{\circ}$ Les propriétés de ce tubercule à l'état frais sont probablement très énergiques, et doivent rivaliser avec celles du Colchique ordinaire. Elles s'émoussent et se perdent par la vétusté.

$4^{\circ} \mathrm{Si}$ les botanistes-médecins de la Renaissance avaient su reconnaître l'identité générique de l'Hermodactylos, de notre Hermodacte officinal et du Colchique ordinaire, on n'aurait pas laissé dormir, jusqu'au commencement de notre siècle, les propriétés du Colchique dans les affections articulaires, propriétés déjà connues des médecins grecs du $\mathbf{v i}^{\mathrm{e}}$ et du viı ${ }^{\mathrm{e}}$ siècle (au moins chez le Colchicum variegatum). 
- $5^{\circ}$ L'Hermodactylus verus de Matthiole (Hermodactylus tuberosus, Salisb., Iris tuberosa, L.) ne saurait être l'Hermodactylos des Grees, ni surtout notre Hermodacte officinal.

6. Le Surugen, ou Hermodactyle à racine longue de Mésué, répond probablement à la forme dactylö̈de du tubercule du Colchicum variegatum, ou de quelque autre espèce orientale.

$7^{\circ}$ On ne saurait déterminer rigoureusement l'espèce de Colchique ì laquelle se rapporte le Surugen à racine ronde et blanche de Mésué. Il est possible que ce soit notre Hermodacte officinal; mais la récolte de ce $S$ urugen est indiquée pour le printemps (1), et celle de notre Hermodacte doit se faire en automne. Peut-être s'agit-il de l'Hermodacte d'Égypte (Colchicum bulbocadioides) dont parle Prosper Alpin.

$8^{\circ}$ Le Surengian d' Avicenne comprend probablement des espèces différentes de Colchique.

$9^{\circ}$ Le Colchicum illyricum est une espèce imaginaire dont le nom doit être rayé des catalogues.

10 L'Hermodacte de Prosper Alpin est le Colchicum bulbocodioides, M. Biebst. (C. agyptiacum, Boiss.) (2).

$11^{\circ}$ Lè tubercule des Colchiques est une base renflée de rameau analogue au plateau des Crocus, et aux pseudo-bulbes de certaines Orehidées. Il répond physiologiquement à certains embryons monocolylédonés; mais ses rapports avec l'embryon des Graminées se bornent à de simples ressemblances.

$12^{\circ}$ Les tubercules de l'Hermodactylus tuberosus sont de vrais rhizomes axillaires, d'abord enveloppés par les bases sacciformes de leurs premières feuilles.

\section{EXPLICATION DES FIGURES.}

Fig. 1. Tubercule d'Hermodacte des officines, vu du côté dorsal : $a$, cicatrice d'insertion de ce tubercule sur celui de l'année précédente : $b$, gemme ou

(1) Une phrase de l'article consacré par Avicenne au Surengian (ou Surugen) semble indiquer que cette plante fleurit au printemps : " Et aperitu imprimis cum aperiuntur flores, " dit l'auteur arabe (ci-dessus, p. 11, note 1). Ceci s'appliquerait mieux au Colchicum bulbocodioides qu'aux colchiques à fleurs automnales.

(2) Serait-ce le Lagia agrestis dont parle Sérapion? (ci-dessus, p. 11, ligne 17) : c'est ce que semblerait indiquer la patrie (Africa) et la propriété de provoquer l'embonpoint chez les femmes. 
bourgeon dorsal : c, cicatrice d'insertion de la tige florifère de l'année précédente.

Fig. 2. Le même tubercule, vu de face : $x$, fossette d'insertion de la tige florifère de l'année.

Fig. 3. Tubercule de Colchicum variegatum, L. dépouillé de sa tunique et vu sur le dos: $a, b, c$, même signification que chez la fig. 1, sauf que la gemme $b$ est ici développée en une tige naissante, dont on n'a conservé que la base. Cette figure et la suivante représentent les objets tels qu'ils sont, à Paris, dans la première quinzaine de décembre.

Fig. 4. Tubercule du Colchicum variegatum L. (forme dactyloïde), revêtu de sa tunique et vu sur le dos ; $a$, cicatrice d'insertion du tubercule sur celui de l'année précédente; $e$, processus en forme d'ongle du tubercule; $c$, base desséchée de la tige florifère de l'automne précédent ; $d$, jeune pousse feuillée de la tige qui vient de fleurir, et qui nait de la base du tubercule.

Fig. 5. Partie inférieure d'un pied d'Hermodactylus tuberosus (Iris tuberosa) pris à l'époque dela floraison, c'est-à-direvers le mois d'avril : $r$, rhizome devenu ligneux, et revêtu d'une écorcebrune; $s$, lambeaux de l'extrémité du tubercule que le bourgeon déchire en se développant; $u$, tige principale et centrale, se terminant par la fleur; $v, v$, deux rameaux secondaires encore stériles, liés chacun à un tubercule qui en forme la base ; $t, t$, deux feuilles squamiformes, scarieuses, percées l'une et l'autre par le tubercule qui naît d'abord à leur aisselle, et se fait jour en les déchirant à leur base ; $y, y$, lambeaux déchirés de la base des feuilles, dont on voit le prolongement vaginiforme en $v, v ; x, x$, bases en cœecum des feuilles dont on voit le limbe linéaire faire saillie hors des gaines $v$, $v$. Au-dessous de ces bases distendues des feuilles, se trouve le tubercule, dont l'extrémité ne tardera pas à poindre à travers la feuille déchirée.

Fig. 6. Deux des tubercules de l'Hermodactylus tuberosus (Iris tuberosa), tels quïils se montrent à Gand, en Belgique, dans les premiers jours de décembre. Il ne reste à la surface de ces branches tubéreuses du rhizome que des vestiges du squelette fibreux des bases de feuilles qui leur avaient d'abord servi d'enveloppe. A leur extrémité $z, z$, on voit une gemme étiolée qui commence à se développer.

Fig. 7. Un des tubercules précédents vu en dessous : $z$, bourgeon terminal : $m$, trace de la ligne d'insertion de la première feuille (disparue) $: n$, trace de la ligne d'insertion de la seconde feuille (également détruite) Ces deux lignes se prolongent sur la portion supérieure du tubercule, de manière à l'embrasser en entier.

Fig. 8. Coupe longitudinale d'un de ces tubercules, pour en faire voir le bourgeon terminal. - Toutes ces figures sont de grandeur naturelle.

Vu, bon à imprimer,

Le directeur de l'École, 



Tubercules du Colchicum variegatum et de L'Hermodactylus tuberosus.

$$
\text { 1- } 4 \text { N.Remond imp.r. des Noyers. 65. Paris. }
$$

